\title{
HESSIANS OF SPECTRAL ZETA FUNCTIONS
}

\author{
K. OKIKIOLU
}

\begin{abstract}
Let $M$ be a compact manifold without boundary. Associated to a metric $g$ on $M$ there are various Laplace operators, for example the De Rham Laplacian on $p$-forms and the conformal Laplacian on functions. For a general geometric differential operator of Laplace type with eigenvalues $0 \leq \lambda_{1} \leq \lambda_{2} \leq \ldots$ we consider the spectral zeta function $Z(s)=\sum_{\lambda_{j} \neq 0} \lambda_{j}^{-s}$. The modified zeta function $\mathcal{Z}(s)=\Gamma(s) Z(s) / \Gamma(s-n / 2)$ is an entire function of $s$. For a fixed value of $s$ we calculate the hessian of $\mathcal{Z}(s)$ with respect to the metric and show that it is given by a pseudodifferential operator $T_{s}=U_{s}+V_{s}$ where $U_{s}$ is polyhomogeneous of degree $n-2 s$ and $V_{s}$ is polyhomogeneous of degree 2 . The operators $U_{s} / \Gamma(n / 2+1-s)$ and $V_{s} / \Gamma(n / 2+1-s)$ are entire in $s$. The symbol expansion of $U_{s}$ is computable from the symbol of the Laplacian. Our analysis extends to describe the hessian of $(d / d s)^{k} \mathcal{Z}(s)$ for any value of $k$.
\end{abstract}

\section{Introduction.}

Let $M$ be an $n$-dimensional smooth compact manifold without boundary. The tensor powers of $\mathbb{R}^{n}$ are $T^{p, q}\left(\mathbb{R}^{n}\right)=\otimes^{p} \mathbb{R}^{n} \otimes \otimes^{q} \mathbb{R}^{n *}$. Suppose $U$ is a vector subspace of $T^{p, q}\left(\mathbb{R}^{n}\right)$ which is invariant under the action of the general linear group $G L\left(\mathbb{R}^{n}\right)$. Writing $\mathcal{P}$ for the principal frame bundle of $M$, we obtain a general tensor bundle $E$ over $M$ by forming the associated bundle

$$
E=\mathcal{P} \times_{G L\left(\mathbb{R}^{n}\right)} U
$$

For example we obtain in this way $T^{p, q}(M)$, the bundle of type- $(p, q)$ tensors on $M$, and $S^{2} M$, the bundle of symmetric $(0,2)$-tensors. Let $\operatorname{End}(E)$ denote the bundle whose fibers are the endomorphisms of the fibers of $E$, so $\operatorname{End}(E)$ can be identified with $E \otimes E^{*}$. Let $N$ denote the rank of $E$, and let $C^{\infty}(E)$ denote the smooth sections of $E$.

For a Riemannian metric $g$ on $M$, let $d V$ denote the canonical volume element in the metric $g$, and $V$ the total volume of $M$. The metric $g$ naturally gives a metric $\langle,\rangle_{g}$ on $E$ which gives an inner product $\left\langle\langle,\rangle_{g}\right.$ on $C^{\infty}(E)$,

$$
\langle\langle\theta, \omega\rangle\rangle_{g}=\int_{M}\langle\theta, \omega\rangle_{g} d V, \quad \theta, \omega \in C^{\infty}(E) .
$$

The metric $g$ also gives rise to the Levi-Civita connection on $M$ with curvature tensor $R$, and the connection on $E$

$$
\nabla=\nabla_{g}: C^{\infty}\left(T^{k} M \otimes E\right) \rightarrow C^{\infty}\left(T^{k+1} \otimes E\right),
$$

which is invariant under scaling, that is for $c>0$ and $\omega \in T^{k}(M \otimes E)$ we have $\nabla_{c g} \omega=\nabla_{g} \omega$.

The author was supported by the National Science Foundation \#DMS-9703329.

Typeset by $\mathcal{A}_{\mathcal{M}} \mathcal{S}-\mathrm{T}_{\mathrm{E}} \mathrm{X}$ 
Now suppose that for each Riemannian metric $g$ we have a differential operator $F=F_{g}: C^{\infty}(E) \rightarrow$ $C^{\infty}(E)$ which is self-adjoint with respect to $\left\langle\langle,\rangle_{g}\right.$, non-negative, scale invariant, and geometric of Laplace type, meaning that

$$
F \omega=V^{2 / n}\left(\sum_{i=1}^{n} \nabla^{2}\left(e_{i}, e_{i}, \omega\right)+\sum_{i, j, k, \ell=1}^{n} R\left(e_{i}, e_{j}, e_{k}, e_{\ell}\right) A\left(\theta^{i}, \theta^{j}, \theta^{k}, \theta^{\ell}, \omega\right)\right)+B \omega
$$

for $\omega \in C^{\infty}(E)$, where $B$ is a constant, $e_{1}, \ldots, e_{n}$, is a pointwise $g$-orthonormal base for $T M$ with dual coframe $\theta^{1}, \ldots, \theta^{n}$, and $A \in C^{\infty}\left(T^{4,0}(M) \otimes \operatorname{End}(E)\right)$ is obtained from some element

$$
\mathcal{A} \in T^{4,0}\left(\mathbb{R}^{n}\right) \otimes U \otimes U^{*}
$$

which is invariant under the natural action of $O\left(\mathbb{R}^{n}\right)$. Indeed, if $\mathcal{O}$ is the $g$-orthonormal frame bundle of $M$, then

$$
T^{4,0}(M) \otimes E \otimes E^{*} \equiv \mathcal{O} \times_{O\left(\mathbb{R}^{n}\right)}\left(T^{4,0}\left(\mathbb{R}^{n}\right) \otimes U \otimes U^{*}\right)
$$

and $\mathcal{A}$ naturally gives rise to a section $A=A_{g}$ of $T^{4,0}(M) \otimes E \otimes E^{*}$. Examples of $F$ include the de Rham Laplacian on forms and the conformal Laplacian.

Write $\Re s$ for the real part of $s \in \mathbb{C}$. Let $\lambda_{1} \leq \lambda_{2} \leq \ldots$ be the eigenvalues of $F$ and set

$$
Z(s)=\sum_{j=1}^{\infty} \lambda_{j}^{-s}
$$

Then $Z(s)$ converges for $\Re s>n / 2$ and extends analytically to a meromorphic function with possible simple poles at

$$
s= \begin{cases}n / 2, n / 2-1, \ldots 1, & \text { for } n \text { even, } \\ n / 2, n / 2-1, \ldots \ldots & \text { for } n \text { odd }\end{cases}
$$

In fact, if $F$ is invertible, then

$$
\mathcal{Z}(s):=\frac{\Gamma(s) Z(s)}{\Gamma(s-n / 2)}
$$

is entire. In general, when $F$ has a kernel of stable dimension $d$, then

$$
\mathcal{Z}_{g}(s)+\frac{d}{\Gamma(s-n / 2) s} \text { is entire. }
$$

and so for two metrics $g$ and $\tilde{g}, \mathcal{Z}_{\tilde{g}}(s)-\mathcal{Z}_{g}(s)$ is entire.

Remark. (1.3) does not hold for general polyhomogeneous pseudodifferential operators of stable dimensional kernel in odd dimensions, since $Z(s)$ may not vanish at the negative integers. See [Ok1] 1.2 for further details.

With $s$ fixed, differentiating $\mathcal{Z}(s)$ twice with respect to the metric one obtains the Hessian of $\mathcal{Z}(s)$ which is a symmetric bilinear form on the space $C^{\infty}\left(S^{2} M\right)$. At the metric $g$,

$$
\operatorname{Hess} \mathcal{Z}(s)(h, h)=\left.\frac{d^{2}}{d \sigma^{2}}\right|_{\sigma=0} \mathcal{Z}_{g+\sigma h}(s) .
$$

Set $\mathbb{N}^{+}=\{1,2, \ldots\}$. 
Theorem 1. For $s \in \mathbb{C}$, there exists a unique symmetric pseudodifferential operator $T_{s}=T_{s}(F)$ : $C^{\infty}\left(S^{2} M\right) \rightarrow C^{\infty}\left(S^{2} M\right)$ such that

$$
\operatorname{Hess} \mathcal{Z}(s)(h, h)=\left\langle\left\langle h, T_{s} h\right\rangle_{g} .\right.
$$

The operator $T_{s}$ is analytic in $s$. For $s \notin n / 2+\mathbb{N}^{+}$, there exist polyhomogeneous pseudodifferential operators $U_{s}$ and $V_{s}$ of degrees $n-2 s$ and 2 respectively such that $T_{s}=U_{s}+V_{s}$. The operators $U_{s}$ and $V_{s}$ are meromorphic in $s$ with simple poles located in $n / 2+\mathbb{N}^{+}$. (The poles of $U_{s}$ and $V_{s}$ cancel in the sum $T_{s}=U_{s}+V_{s}$, but the symbol expansion of $T_{s}$ for $s \in n / 2+\mathbb{N}^{+}$involves logarithmic terms.) For general $s$, the polyhomogeneous symbol expansion of $U_{s}$ is computable from the complete symbol of the operator $F$. In particular, there is a simple algorithm to compute the term $u_{s}$ of homogeneity $n-2 s$. Furthermore, we can differentiate in s to obtain

$$
\operatorname{Hess}(d / d s)^{k} \mathcal{Z}(s)(h, h)=\left\langle\left\langle h,(d / d s)^{k} T_{s} h\right\rangle_{g},\right.
$$

and the principal symbol of $(d / d s)^{k} U_{s}$ is equal to the leading order term of $(d / d s)^{k} u_{s}$ (provided this does not vanish identically).

Theorem 2. The symbol $u_{s}$ from Theorem 1, can be computed as follows. Write $F^{\prime}=\left.(d / d \sigma)\right|_{\sigma=0} F(g+$ $\sigma h)$ and let $x \in M$. Take coordinates on $M$ which are orthonormal at the point $x$, and take a local trivialization of $E$ on a neighborhood of $x$ to obtain coordinates for $E$. Suppose that in these coordinates, the operator $F^{\prime}$ is given at the point $x$ by

$$
F^{\prime}=\sum_{\alpha, \beta, i, j} A_{\alpha, \beta}^{i j}\left(\partial_{w}^{\alpha} h_{i j}(x)\right) \partial_{w}^{\beta},
$$

where $\alpha$ and $\beta$ are multiindices and $A_{\alpha, \beta}^{i j}$ is an $N \times N$ real valued matrix. Set

$$
S=s-n / 2,
$$

write I for the identity operator on $E_{x}$, and set

$$
C(s)=\left(\frac{1}{4 \pi}\right)^{n / 2} \frac{\Gamma(-S+1)^{2}}{\Gamma(-2 S+2)}
$$

Then at $(x, \xi) \in T^{*} M$, the value of $u_{s}(x, \xi) \in \operatorname{End}\left(S^{2} M\right)_{x}$ is given by

$$
\left(u_{s}(x, \xi) h\right)_{i j}=V^{(2 s-n) / n} C(s) \sum_{\substack{|\alpha|+|\beta|=2 \\|\gamma|+|\delta|=2 \\ k, \ell}} u_{s}\left(\partial^{\alpha}, \partial^{\beta}, \partial^{\gamma}, \partial^{\delta}, x, \xi\right) \operatorname{trace}\left(A_{\alpha, \beta}^{i j}(x) A_{\gamma, \delta}^{k \ell}(x)\right) h_{k \ell},
$$

where the terms $u_{s}\left(\partial^{\alpha}, \partial^{\beta}, \partial^{\gamma}, \partial^{\delta}, x, \xi\right)$ are given as follows:

$$
\begin{aligned}
& u_{s}\left(\partial_{j} \partial_{k}, I, \partial_{p} \partial_{q}, I, x, \xi\right)=4\left(4 S^{2}-1\right) \xi_{j} \xi_{k} \xi_{p} \xi_{q}|\xi|^{n-2 s-4} . \\
& u_{s}\left(\partial_{j}, \partial_{k}, \partial_{p} \partial_{q}, I, x, \xi\right)=-2\left(4 S^{2}-1\right) \xi_{j} \xi_{k} \xi_{p} \xi_{q}|\xi|^{n-2 s-4} .
\end{aligned}
$$




$$
\begin{gathered}
u_{s}\left(\partial_{j}, \partial_{k}, \partial_{p}, \partial_{q}, x, \xi\right)=\left(4 S^{2}+2 S-2\right) \xi_{j} \xi_{k} \xi_{p} \xi_{q}|\xi|^{n-2 s-4}-(2 S-1) \delta_{k q} \xi_{j} \xi_{p}|\xi|^{n-2 s-2} \\
u_{s}\left(I, \partial_{j} \partial_{k}, \partial_{p} \partial_{q}, I, x, \xi\right)=\left(4 S^{2}-2 S\right) \xi_{j} \xi_{k} \xi_{p} \xi_{q}|\xi|^{n-2 s-4}+(2 S-1) \delta_{j k} \xi_{p} \xi_{q}|\xi|^{n-2 s-2} . \\
\begin{array}{r}
u_{s}\left(I, \partial_{j} \partial_{k}, \partial_{p}, \partial_{q}, x, \xi\right)= \\
+\left(2 S^{2}+S-1\right) \xi_{j} \xi_{k} \xi_{p} \xi_{q}|\xi|^{n-2 s-4} \\
+(S-1 / 2)\left(-\delta_{j k} \xi_{p} \xi_{q}+\delta_{j q} \xi_{k} \xi_{p}+\delta_{k q} \xi_{j} \xi_{p}\right)|\xi|^{n-2 s-2}
\end{array}
\end{gathered}
$$

$$
\begin{aligned}
& u_{s}\left(I, \partial_{j} \partial_{k}, I, \partial_{p} \partial_{q}, x, \xi\right)= \\
& \left(S^{2}+S\right) \xi_{j} \xi_{k} \xi_{p} \xi_{q}|\xi|^{n-2 s-4}+\frac{1}{2}(S-1) \delta_{j k} \xi_{p} \xi_{q}|\xi|^{n-2 s-2}+\frac{1}{2}(S-1) \xi_{j} \xi_{k} \delta_{p q}|\xi|^{n-2 s-2} \\
& -\frac{S}{2}\left(\delta_{j p} \xi_{k} \xi_{q}+\delta_{k q} \xi_{j} \xi_{p}+\delta_{j q} \xi_{k} \xi_{p}+\delta_{k p} \xi_{j} \xi_{q}\right)|\xi|^{n-2 s-2}+\frac{1}{4}\left(\delta_{j k} \delta_{p q}+\delta_{j p} \delta_{k q}+\delta_{j q} \delta_{k p}\right)|\xi|^{n-2 s}
\end{aligned}
$$

We refer to the operator $T_{s}$ in Theorem 1, as the operator corresponding to Hess $\mathcal{Z}(s)$. At a metric $g$ which is critical for $T_{s}$, the eigenvalues of $T_{s}$ can determine whether $g$ is a local maximum, minimum or saddle point. Computation of the principal symbol of $T_{s}$ gives at least some information about the type of critical point, for example if it changes sign, one can say for sure that $g$ is a saddle point, while if it is positive, one can generally determine that $g$ has finite index. The operators $U_{s}, V_{s}$ are only well defined up to the addition (respectively subtraction) of a smoothing operator which is analytic in $s$. They are however canonically defined on the level of symbol expansions because in general they have degrees which differ by a non-integer. It should be noted that while the symbol expansion of $U_{s}$ is locally computable, the expansion of $V_{s}$ generally involves global quantities. Theorem 1 sheds light on the question of [GS] concerning how many derivatives of an invariant must be computed to obtain a local expression. We see that for low values of $s$, it takes two derivatives of a zeta invariant to obtain an expression whose "leading order" is local. Indeed, in general $u_{s}$ is the principal symbol of $T_{s}$ when $\Re s<n / 2-1$ and this is local, while $v_{s}$ is the principal symbol when $\Re s>n / 2-1$. Of particular interest in this discussion is the zeta-regularized determinant of $F$, which is defined to be $e^{-Z^{\prime}(0)}$. The quantities $u(0)$ and $\left.(d / d s)\right|_{s=0} u_{s}$ usually control the type of critical points possible for the determinant. The picture may become complicated at values of $s$ for which $u_{s}$ or $v_{s}$ vanishes. At these values, the principal symbol of $T_{s}$ is given by the highest degree non-vanishing term in the symbol of $U_{s}$ or $V_{s}$, or if these terms have the same degree it is given by the sum. For example, in [Ok2] we will actually compute the principal symbol of $V_{s}$ for the family of operators in (1.9). We will see cases when $V_{s}$ has order zero, and $u_{s}$ is the principal symbol of $T_{s}$ for $\Re s<n / 2$. We also remark that one can formulate a slightly more abstract but more basic version of Theorem 1, which does not mention metrics or geometric operators. Indeed, fix a tensor bundle over $M$, and consider the space of elliptic, non-negative second order differential operators with isotropic principal symbols. Now consider the space of coefficients of such operators and compute the Hessian of the zeta function with respect to variation of the coefficients. Then this bilinear form on the space of coefficients is given by a pseudodifferential operator, in a similar fashion to Theorem 1. Theorem 1 then follows from the more abstract formulation by expressing the coefficients of a Laplace type operator in terms of the metric.

In this paper we prove Theorems 1 and 2. Although similar results hold for geometric elliptic operators of more general form, we will not deal with greater generality here. For us the main goal is to understand the behavior of zeta functions for specific geometric operators. In two further papers 
[Ok2] and $[\mathrm{OW}]$, we carry out specific calculations. In [Ok2], we explicitly compute $u_{s}$ for the family of Laplacians

$$
F=V^{2 / n}\left(\Delta+c_{1} \mu S\right)+c_{2} I
$$

where $\Delta$ is the (positive) Laplace-Beltrami operator, $S$ is the scalar curvature, $I$ is the identity operator, $\mu=(n-2) /(4(n-1))$, and $c_{1}, c_{2}$ are arbitrary constants which render $F$ non-negative. This family contains both the Laplace-Beltrami operator and the conformal Laplacian $\left(c_{1}=1, c_{2}=0\right)$, and we are in fact able to compute the principal symbol of $V_{s}$ which is non-local. We go on to make conclusions about the critical points of $Z(s)$ for all values of $s$. For large values of $s, Z(s)$ should be dominated by the lowest eigenvalues, and one sees consequenses of this in the results. In joint work with C. Wang, [OW], we compute $u_{s}$ for the de Rham Laplacian. Theorem 2 (a)-(f) gives the symbol $u_{s}$ needed for the applications [Ok2] and [OW]. The general formula for $u_{s}$ is given in Lemma 5.1. We do not give here explicit formulas for the complete symbol expansions of $U_{s}$ and $V_{s}$, although the symbol expansion of $U_{s}$ can be obtained from (5.10) or Lemmas 6.1,6.5, and the symbol expansion of $V_{s}$ has contributions from three terms, firstly (4.5), secondly (4.16) whose symbol expansion can be computed from (4.18), and thirdly (4.8) whose symbol expansion is computed in Lemmas 6.1, 6.5.

This work is motivated by a growing list of results [OPS1], [BCY], [CY], [Br], [Ri], [Ok1], [Mo1], [Mo2], where the authors take $F$ to be the conformal Laplacian or Laplace-Beltrami operator, and identify local or global maximal or minimal metrics for the functional $\log \operatorname{det} F$ or $Z(s)$, either within a conformal class, or among all smooth metrics. Papers [OPS2], [OPS3] and [CGY] contain applications of some of those results. It emerges that in some situations only maxima occur while in others only minima occur. In [Ok2] and [OW] we will address the questions of when it is possible for maxima to occur, when it is possible for minima to occur, when neither can happen, and when one might hope to find a convexity argument to prove global results.

Acknowledgements. The author would like to thank the referees for their careful and extensive comments on earlier versions of this manuscript. In addition, the author would also like to thank the following institutions for support: ESI, IAS, IHES, MSRI, Princeton University.

We will now indicate briefly what is involved in the proof of Theorems 1 and 2. One can use the parametrix for the heat kernel or the resolvant of the Laplacian to prove the result. Technically the work involved is comparable for the two methods, and each method has its advantages. The heat kernel method has the advantage of yeilding completely explicit formulas for the symbol expansion of $U_{s}$, and so we take this approach. We fix a metric $g$, and take a smooth one-parameter deformation $\tilde{g}(\sigma)$ with $\tilde{g}(0)=g$. Using the Melin transform to write $\mathcal{Z}_{\tilde{g}(\sigma)}(s)$ in terms of the heat kernel, we can differentiate to compute the second derivative of $\mathcal{Z}_{\tilde{g}}(s)$ with respect to $\sigma$ at $\sigma=0$, see Lemma 3.1. The main term we need to study is the one giving rise to the operator $U_{s}$, which is the last term on the right hand side of (3.1). Writing $F^{\prime}=\left(\partial F_{\tilde{g}(\sigma)} / \partial \sigma\right)(0)$, it equals

$$
\frac{1}{\Gamma(s-n / 2)} \operatorname{trace} \iint_{u+v<1}(u+v)^{s} F^{\prime} e^{-u F} F^{\prime} e^{-v F} d u d v
$$

Before proceeding, we give a list of the steps in the proof to help the reader navigate. The steps in this guide will usually be referenced when they occur in the proofs that follow.

Table 1.1: Steps of the Proof. This table lists the steps which are needed to go from the expression (1.10) to the symbol expansions of the operators $U_{s}$ and $V_{s}$. They are not listed here in the order they 
occur in the proof, and some of the steps need to be repeated more than once in the proof. Steps 1-8 are entirely trivial while Steps 9-16 represent the analytic details, with Steps 13-16 being the more significant or subtle. On the other hand, one has to pay a little attention to steps 7, 8 and 9, because they change the nature of the expression being considered.

Step 1: The steps just carried out produced a sum of terms. Consider each term separately.

Step 2: At a previous stage, the expression was decomposed as a sum and each term was considered separately. Reverse this by summing up the contributions from each term.

Step 3: Introduce a partition of unity to reduce to coordinate charts over which $E$ is trivial.

Step 4: Work in geodesic normal coordinates.

Step 5: Write $h=\tilde{g}^{\prime}(0)$ in components.

Step 6: Write $F^{\prime}$ in components.

Step 7: The expression has the form " $\iint\left((u+v)^{s}\right.$ — dudv". Restrict attention to the term "_ " in the integrand.

Step 8: Reverse Step 8 by reinstating the factor $(u+v)^{s}$ and the uv integration.

Step 9: Identify an operator $\tilde{T}$ (by its kernel) so that the expression has the form $\langle\langle h, \tilde{T} h\rangle$.

Step 10: Write the trace of an operator as the integral of the kernel over the diagonal.

Step 11: Make the standard small time expansion of the heat kernel.

Step 12: Make a Taylor expansion of each term in a given variable.

Step 13: Collect together terms of the same "order".

Step 14: Exchange uv integration in the expression with another limiting process such as taking the operator trace, a Fourier transform or integration in other variables.

Step 15: Compute the symbol of the operator from its kernel by taking the Fourier transform. Step 16: The symbol has the form $\iint\left((u+v)^{s} \_d u d v "\right.$. Carry out the uv integration to see the asymptotic behavior in the cotangent variable $\xi$.

The simplified scalar case: We wish to write (1.10) in terms of $h=(d \tilde{g} / d \sigma)(0)$. Let us first pretend for the sake of clarity that $F^{\prime}$ is just multiplication by the smooth function $\phi=h_{i j} A^{i j}$, where $A^{i j}$ is a smooth tensor field. In doing so we will be ignoring some technicalities which arise when $A$ is a differential operator, but let us concentrate here on the main point. This eliminates the need for Steps 5, 6. We now wish to compute

$$
\frac{1}{\Gamma(s-n / 2)} \operatorname{trace} \iint_{u+v<1}(u+v)^{s} \phi e^{-u F} \phi e^{-v F} d u d v .
$$

Steps 14, 10: Writing $K\left(e^{-t F}, x, y\right)$ for the integral kernel of $e^{-t F}$, this is equal to

$$
\frac{1}{\Gamma(s-n / 2)} \iint_{u+v<1}(u+v)^{s} \iint_{M \times M} \phi(x) K\left(e^{-u F}, x, y\right) \phi(y) K\left(e^{-v F}, y, x\right) d V(x) d V(y) d u d v
$$

Steps 14, 9: We see that this equals

$$
\left\langle\left\langle\phi, \tilde{T}_{s} \phi\right\rangle\right\rangle
$$

where $\tilde{T}_{s}$ is the operator on functions with

$$
\left(\tilde{T}_{s} \phi\right)(x)=\int_{M} K_{s}(x, y) \phi(y) d V(y)
$$


where

$$
K_{s}(x, y)=\frac{1}{\Gamma(s-n / 2)} \iint_{u+v<1}(u+v)^{s} K\left(e^{-u F}, x, y\right) K\left(e^{-v F}, y, x\right) d u d v .
$$

To express this operator as a psuedodifferential operator, we compute its symbol by taking the Fourier transform. Here, we focus just on the leading order term in this symbol.

Steps 3, 1, 4: Fix a point $x \in M$ and work in normal coordinates $w$ of $y$ about $x$.

Steps 7, 11, 13: For this discussion, let us assume $V=1$, and restrict attention to the leading order term of the heat kernel $K\left(e^{-t F}, x, y\right)$, which a constant multiple of, $t^{-n / 2} e^{-|w|^{2} / 4 t}$. Approximately then we have

$$
K\left(e^{-u F}, x, y\right) K\left(e^{-v F}, y, x\right) \approx C(u v)^{-n / 2} e^{-|w|^{2}(1 / 4 u+1 / 4 v)} .
$$

Step 15: Since $\sqrt{g(x)}=1$, up to leading order, the symbol of the right hand side of (1.16) is obtained by taking the Fourier transform in $w$. This yields a constant multiple of,

$$
(u+v)^{-n / 2} e^{-|\xi|^{2} u v /(u+v)} .
$$

Steps 14, 8: From this, we see that at $x$, the symbol of $\tilde{T}_{s}$, whose kernel is given by (1.15), is approximately

$$
\frac{1}{\Gamma(s-n / 2)} \iint_{u+v<1}(u+v)^{s-n / 2} e^{-|\xi|^{2} u v /(u+v)} d u d v
$$

Step 16: Substituting $\tau=u /(u+v)$ and $T=|\xi|^{2}(u+v)$, this is

$$
\frac{|\xi|^{n-2 s-4}}{\Gamma(s-n / 2)} \int_{T<|\xi|^{2}} \int_{\tau=0}^{1} T^{s-n / 2+1} e^{-T \tau(1-\tau)} d \tau d T .
$$

If we replace the interval of integration by $0<T<\infty$, we get

$$
\frac{|\xi|^{n-2 s-4}}{\Gamma(s-n / 2)} \int_{T=0}^{\infty} \int_{\tau=0}^{1} T^{s-n / 2+1} e^{-T \tau(1-\tau)} d \tau d T=\frac{\Gamma(n / 2-s-1) \Gamma(n / 2-s+1)|\xi|^{n-2 s-4}}{\Gamma(n-2 s-2)}
$$

This gives the principal symbol of $U_{s}$. The reason it has degree $n-2 s-4$ instead of $n-2 s$ as stated in Theorem 1, is because we have assumed that $F^{\prime}$ is just multiplication by the function $\phi=h_{i j} A^{i j}$, whereas in general it may involve derivatives up to order 2. Since $F^{\prime}$ occurs twice in (1.10), this accounts for the four missing derivatives. To compute (1.19) we need to subtract from (1.20) the part of the integral on the left hand side over the interval $|\xi|^{2}<T<\infty$. But by steepest descent, there is an expansion as $T \rightarrow \infty$ of the form

$$
\int_{\tau=0}^{1} e^{-T \tau(1-\tau)} d \tau \sim 2 T^{-1}+C_{2} T^{-2}+C_{3} T^{-3}+\ldots
$$

Hence as $|\xi| \rightarrow \infty$,

$$
\begin{aligned}
\frac{|\xi|^{n-2 s-4}}{\Gamma(s-n / 2)} \int_{T>|\xi|^{2}} T^{s-n / 2+1} & \int_{\tau=0}^{1} e^{-T \tau(1-\tau)} d \tau d T \\
& \sim \frac{-1}{\Gamma(s-n / 2)}\left(\frac{2|\xi|^{-2}}{s-n / 2+1}+\frac{C_{2}|\xi|^{-4}}{s-n / 2}+\frac{C_{3}|\xi|^{-6}}{s-n / 2-1}+\ldots\right) .
\end{aligned}
$$


This is a symbol of order -2 . The poles, which occur at $s=n / 2+1, n / 2+2, \ldots$, cancel with the poles of (1.20) and contribute logarithmic terms to the symbol of $\tilde{T}_{s}$ at these values of $s$.

The general case: Now we modify this basic calculation to obtain Theorem 2 on the tensor bundle $E$.

Step 5: Suppose that $h$ is supported on a small neighborhood $\Omega$ of a point $x$ on which we take coordinates.

Step 6: Write $F^{\prime}$ in components on $\Omega$ as

$$
F^{\prime}=\sum_{\alpha, \beta, i, j} A_{\alpha, \beta}^{i j}\left(\partial_{w}^{\alpha} h_{i j}\right) \partial_{w}^{\beta},
$$

so that (1.10) becomes

$$
\sum_{\substack{\alpha, \beta, \gamma, \delta \\ i, j, k, \ell}} \frac{1}{\Gamma(s-n / 2)} \operatorname{trace} \iint_{u+v<1}(u+v)^{s} A_{\alpha \beta}^{i j}\left(\partial^{\alpha} h_{i j}\right) \partial^{\beta} e^{-u F} A_{\gamma \delta}^{k \ell}\left(\partial^{\gamma} h_{k \ell}\right) \partial^{\delta} e^{-v F} d u d v .
$$

In fact, because we are only interested in the leading order term in the symbol, we can restrict the sum in (1.23) to those multiindices with $|\alpha+\beta|=|\gamma+\delta|=2$.

Steps 1, 14, 10: We will analyze each term in the sum (1.23) separately. We write

$$
\begin{aligned}
& \frac{1}{\Gamma(s-n / 2)} \operatorname{trace} \iint_{u+v<1}(u+v)^{s} A_{\alpha \beta}^{i j}\left(\partial^{\alpha} h_{i j}\right) \partial^{\beta} e^{-u F} A_{\gamma \delta}^{k \ell}\left(\partial^{\gamma} h_{k \ell}\right) \partial^{\delta} e^{-v F} d u d v \\
= & \frac{(-1)^{|\alpha+\gamma|}}{\Gamma(s-n / 2)} \iint_{u+v<1}(u+v)^{s} \iint_{M \times M}\left(\partial^{\alpha} h_{i j}\right)(x) E(u, v, x, y)\left(\partial^{\gamma} h_{k \ell}\right)(y) d V(x) d V(y) d u d v
\end{aligned}
$$

where

$$
E(u, v, x, y)=\operatorname{trace} A_{\alpha \beta}^{i j}(x)\left(\partial_{x}^{\beta} K\left(e^{-u F}, x, y\right)\right) A_{\gamma \delta}^{k \ell}(y)\left(\partial_{y}^{\delta} K\left(e^{-v F}, y, x\right)\right) .
$$

Steps 14, 9: This has the form

$$
\left\langle\left\langle h_{i j}, T_{s, i j}^{k \ell} h_{k \ell}\right\rangle\right\rangle
$$

where $T_{s, i j}^{k \ell}$ is the operator on scalar valued functions whose kernel to leading order is

$$
\frac{(-1)^{|\alpha+\gamma|}}{\Gamma(s-n / 2)} \iint_{u+v<1}(u+v)^{s} \partial_{x}^{\alpha} \partial_{y}^{\gamma} E(u, v, x, y) d u d v
$$

(We integrated by parts to obtain this. Derivatives falling on the volume factors $\sqrt{g(x)}$ and $\sqrt{g(y)}$ yield lower order terms.)

Steps 11, 13: To compute the principal symbol of the kernel in (1.27), we restrict attention the leading order term of the heat kernel $K\left(e^{-t F}, x, y\right)$, which is a constant multiple of, $t^{-n / 2} e^{-|d(x, y)|^{2} / 4 t} I$, where $d(x, y)$ is the distance from $x$ to $y$. This enables us to replace (1.27) by

$$
\begin{aligned}
& \frac{(-1)^{|\beta+\gamma|} \operatorname{trace} A_{\alpha \beta}^{i j}(x) A_{\gamma \delta}^{k \ell}(x)}{\Gamma(s-n / 2)} \\
& \quad \times \iint_{u+v<1}(u+v)^{s}(u v)^{-n / 2}\left(\partial_{y}^{\alpha} \partial_{y}^{\gamma}\left(\left(\partial_{y}^{\beta} e^{-|d(x, y)|^{2} / 4 u}\right)\left(\partial_{y}^{\delta} e^{-|d(x, y)|^{2} / 4 v}\right)\right)\right) d u d v .
\end{aligned}
$$


Here, we are using the fact that to leading order,

$$
\left.\partial_{x}^{\alpha} e^{-d^{2}(x, y) / 4 u}\right|_{x=0} \sim(-1)^{|\alpha|} \partial_{y}^{\alpha} e^{-d^{2}(x, y) / 4 u}
$$

Step 4: At this point we fix the point $x$ and work in geodesic normal coordinates on $\Omega$ centered at $x$. (The coordinates used in (1.23) to reduce to a scalar problem may be arbitrary.)

Steps 14, 15, 16: Taking the Fourier transform of (1.28) in the normal coordinates of $y$ as in (1.17)(1.20), yields the degree $n-2 s$ term in the symbol of $T_{s, i j}^{k \ell}$ of (1.26). Carrying out the calculation for explicit choices of $(\alpha, \beta, \gamma, \delta)$ gives rise to the quantities in Theorem 2, (a)-(f).

The rest of the paper is organized as follows. In Section 3, we derive variation formulas for $\mathcal{Z}(s)$ to write the Hessian of $\mathcal{Z}(s)$ in terms of derivatives of $F$. In Section 4 , we use basic estimates on the heat kernel to reduce the problem of studying the Hessian to that of studying (1.10) (equivalently (4.19)). In order to analyze the operator associated to (1.10), we will need to make a double decomposition of the integral kernel, one stage to extract the polyhomogeneous expansion of the symbol of $U_{s}$, and the other to extract the polyhomogeneous expansion of $V_{s}$. In Section 5, we extend the above sketch to obtain the expansion of the symbol of $U_{s}$ and use this to deduce Theorem 2. However, we leave the rigorous estimates on errors to Section 6 where we prove Theorem 1 by carrying out the double decomposition in detail. Formula (1.5) then requires no proof because it follows directly from (1.4), the fact that $T_{s}$ is an analytic family of operators, and the standard fact that if the kernel of $F$ has stable dimension then $\mathcal{Z}_{g}(s)$ is smooth on $\mathcal{M} \times \mathbb{C}$.

2. Background and conventions. Before commencing with the proof of Theorem 1 , we discuss in more detail the Hessian of the function $\mathcal{Z}(s)$ and we fix the conventions which will be assumed concerning polyhomogeneous pseudodifferential operators ( $\Psi$ DOs). Let $\mathcal{M}$ denote the space of Riemannian metrics on $M$. Define the Sobolev space $H^{r}\left(S^{2} M\right)$ to be the completion of $C^{\infty}\left(S^{2} M\right)$ in the norm \|\|$_{r}$ defined by

$$
\|h\|_{r}=\sum_{s \leq r}\left\langle\left\langle\nabla^{s} h, \nabla^{s} h\right\rangle\right\rangle_{g}^{1 / 2}
$$

where $g$ is a fixed but arbitrary smooth metric. The Sobolev imbedding theorem shows that for $r>n / 2$, $H^{r}\left(S^{2} M\right)$ is contained in the space $C\left(S^{2} M\right)$ of continuous sections of $S^{2} M$. The smooth topology on $\mathcal{M}$ is the Frechet space topology coming from the collection of all the norms \|\|$_{r}$. For $r>n / 2$, let $\mathcal{M}^{r}$ denote the closure of the space of smooth metrics on $M$ in the space $H^{r}\left(S^{2} M\right)$. For a compact subset $U \subset \mathbb{C}$, let $\operatorname{Hol}(U)$ be the space of holomorphic functions on $U$ with the supremum norm.

Proposition. Suppose $m, r \in \mathbb{N}$ with $r>m+n / 2+1$, and $U \subset\{s \in \mathbb{C}: \Re s>m+n / 2+1-r\}$. Then the map $g \rightarrow \mathcal{Z}_{F_{g}}$ is an $m$-times continuously differentiable function from $\mathcal{M}^{r}$ to $\operatorname{Hol}(U)$.

The proof is given in [Ok3]. Using it, we obtain the Hessian of $(d / d s)^{k} \mathcal{Z}(s)$ as a bilinear form on $H^{r}\left(S^{2} M\right)$, provided $r>n / 2+3-\min \{(0, \Re s)\}$. This restricts to a bilinear form on $C^{\infty}\left(S^{2} M\right)$. We should remark that the Hessian defined above Theorem 1 is computed using the local linear space structure of $\mathcal{M}$, that is using the flat connection on $\mathcal{M}$. It is possible to take other connections on $\mathcal{M}$. For a sufficiently regular function $F: \mathcal{M} \rightarrow \mathbb{C}$ and a smooth curve $\tilde{g}(\sigma)$ in $\mathcal{M}$, the derivative $D F$ is the linear form on $C^{\infty}\left(S^{2} M\right)$ defined by

$$
D F\left(\frac{\partial \tilde{g}}{\partial \sigma}\right)=\frac{\partial F(\tilde{g}(\sigma))}{\partial \sigma}
$$


The Hessian of $F$ relative to a connection $\nabla$ on $\mathcal{M}$ is given at $g \in \mathcal{M}$ by

$$
\operatorname{Hess} F\left(\frac{\partial \tilde{g}}{\partial \sigma}, \frac{\partial \tilde{g}}{\partial \sigma}\right)=\frac{\partial^{2} F(\tilde{g}(\sigma))}{\partial \sigma^{2}}-D F\left(\nabla\left(\frac{\partial \tilde{g}}{\partial \sigma}, \frac{\partial \tilde{g}}{\partial \sigma}\right)\right)
$$

We will prove Theorem 1 when the Hessian is defined relative to the flat or intrinsic connection on $\mathcal{M}$. The intrinsic connection on $\mathcal{M}$ is the Levi-Civita connection coming from the intrinsic metric defined as follows. For $u, v \in C^{\infty}\left(S^{2} M\right)$, at the point $g \in \mathcal{M}$,

$$
G_{\text {intrinsic }}(u, v)=\left\langle\langle u, v\rangle_{g} .\right.
$$

Then

$$
\left(\nabla\left(\frac{\partial \tilde{g}}{\partial \sigma}, \frac{\partial \tilde{g}}{\partial \sigma}\right)\right)_{i j}=\frac{\partial^{2} \tilde{g}_{i j}}{\partial \sigma^{2}}-\tilde{g}^{k \ell} \frac{\partial \tilde{g}_{i k}}{\partial \sigma} \frac{\partial \tilde{g}_{\ell j}}{\partial \sigma}+\frac{1}{2} \tilde{g}^{k \ell} \frac{\partial \tilde{g}_{k \ell}}{\partial \sigma} \frac{\partial \tilde{g}_{i j}}{\partial \sigma}-\frac{1}{4} \tilde{g}_{i j} \tilde{g}^{k \ell} \tilde{g}^{p q} \frac{\partial \tilde{g}_{\ell p}}{\partial \sigma} \frac{\partial \tilde{g}_{k q}}{\partial \sigma} .
$$

Now we give a summary of some facts we will need about polyhomogeneous $\Psi$ DOs. For further details the reader is referred to [Tr] and [Se]. If $U \subset \mathbb{R}^{n}$ is open and $m \in \mathbb{R}$, a function $q \in C^{\infty}(U \times$ $\mathbb{R}^{n}, T^{1,1}\left(\mathbb{R}^{N}\right)$ ) is an $N \times N$-matrix valued symbol of order $m$ (and type $(0,1)$ ), if for all multiindices $\alpha, \beta$ and compact sets $K \subset U$,

$$
\left|\partial_{x}^{\alpha} \partial_{\xi}^{\beta} q(x, \xi)\right| \leq C(1+|\xi|)^{m-|\beta|},
$$

where $C=C(\alpha, \beta, K)$ and $|\cdot|$ is a norm on $T^{1,1}\left(\mathbb{R}^{N}\right)$. The symbol $q$ has order $-\infty$ if it has order $m$ for all $m \in \mathbb{R}$. The symbol $q$ is polyhomogeneous symbol of degree $\mu \in \mathbb{C}$ if there exists a sequence of functions $q_{j} \in C^{\infty}\left(\Omega \times\left\{\xi \in \mathbb{R}^{n}: \xi \neq 0\right\}, T^{1,1}\left(\mathbb{R}^{N}\right)\right), j=0,1,2, \ldots$, which are homogeneous;

$$
q_{j}(x, r \xi)=r^{\mu-j} q_{j}(x, \xi), \quad r>0,
$$

such that

$$
q(x, \xi) \sim q_{0}(x, \xi)+q_{1}(x, \xi)+\ldots, \quad \text { as }|\xi| \rightarrow \infty .
$$

By this we mean that if we introduce a cut-off function $\phi \in C^{\infty}([0, \infty))$ supported on $[1 / 2, \infty)$ with $\phi=1$ on $[1, \infty)$, then

$$
q(x, \xi)-\phi(|\xi|) \sum_{j=0}^{J-1} q_{j}(x, \xi) \text { is a symbol of order } \Re \mu-J
$$

(Where $\Re \mu$ is the real part of $\mu$.)

Now suppose $\pi: E \rightarrow \Omega$ is a rank $N$ vector bundle and $Q: C^{\infty}(E) \rightarrow C^{\infty}(E)$ is a linear operator. Then $Q$ is a $\Psi$ DO of order $m$ if whenever we can trivialize $E$ over a coordinate neighborhood thus identifying it with $\left(U \subset \mathbb{R}^{n}\right) \times \mathbb{R}^{N}$, then there is a symbol $q$ of order $m$ such that

$$
Q h(x)=\frac{1}{(2 \pi)^{n}} \int_{\mathbb{R}^{n}} \int_{M} e^{i(x-y) \cdot \xi} q(x, \xi) h(y) d y d \xi .
$$

for $x$ in $U$ and $h$ supported on a compact subset of $U$. The principal symbol is then a section of the pull back of the bundle $E \otimes E^{*}$ to the cotangent bundle of $M . Q$ is polyhomogeneous of degree $\mu$ if 
and only if for all trivializations of $E$, the symbol $q$ of $Q$ is polyhomogeneous of degree $\mu$. We remark that from this it follows that there is a smooth kernel $k$ on $M \times M \backslash\{(x, x)\}$ such that

$$
Q h(x)=\int_{M} k(x, y) h(y) d y
$$

whenever $x$ is not in the support of $h$. Conversely, if the operator $Q: C^{\infty}(E) \rightarrow C^{\infty}(E)$ is given by a smooth kernel away from the diagonal then it is a $\Psi D O$ of order $m$ provided (2.3) holds on coordinate charts of small diameter. If the operator $Q$ is given by a kernel which is smooth everywhere, it is called a smoothing operator.

When the manifold $M$ is equipped with a Riemannian metric $g$, it is convenient to use the density $d V$ to define the kernel $K(Q, x, y)$ of the operator $Q$, so

$$
Q h(x)=\int_{M} K(Q, x, y) h(y) d V(y)
$$

With this convention, the symbol $q(x, \xi)$ of $Q$ in a given coordinate system is obtained by taking the Fourier transform of $K(Q, x, y) d V(y)$ :

$$
q(x, \xi)=\int K(Q, x, y) \chi(y) e^{i(y-x) \cdot \xi} d V(y)
$$

Here, $\chi$ is a cut off function equal to one on a neighborhood of $x$ and supported in the coordinate chart. The symbol $q$ is well defined up to a symbol of order $-\infty$ arising from the choice of $\chi$. In particular, if $Q$ is polyhomogeneous symbol expansion is well defined at $x$ in the given coordinate system. It will generally be convenient to compute the metric-modified symbol

$$
q(x, \xi)=\int K(Q, x, y) \chi(y) e^{i(y-x) \cdot \xi} d y
$$

rather than the true symbol. The two are of course very simply related.

Suppose now that we choose a smooth function $w: \Omega \times \Omega \rightarrow \mathbb{R}^{n}$ such that for $x$ fixed, $w(x, y)$ defines geodesic normal coordinates of $y$ centered at $x$. Then $(x, y) \rightarrow(x, w)$ defines a smooth diffeomorphism from a neighborhood of the diagonal in $\Omega \times \Omega$ into $\Omega \times \mathbb{R}^{n}$. It follows from [Hö] that (2.3) can be replaced by

$$
Q h(x)=\frac{1}{(2 \pi)^{n}} \int_{\mathbb{R}^{n}} \int_{M} e^{i w \cdot \xi} \tilde{q}(x, \xi) h(y(x, w)) d w d \xi,
$$

whenever there is a set of small diameter in $M$ which contains both the support of $h$ and the point $x$. Here, $\tilde{q}$ is a symbol of order $m$ if we express $x$ in coordinates.

If $Q_{s}: C^{\infty}(E) \rightarrow C^{\infty}(E)$ is a family of $\Psi$ DOs depending on a parameter $s \in \mathcal{O} \subset \mathbb{C}$, then we say that $Q_{s}$ is a analytic family of polyhomogeneous $\Psi D O s$ if $Q_{s}$ has degree $\mu(s)$ where $\mu$ is analytic on $\mathcal{O}$, and on any local trivialization of $E, Q_{s}$ has symbol $q(s, x, \xi)$ which is smooth in $(s, x, \xi)$ analytic in $s$, and satisfies the estimates

$$
\left|\partial_{x}^{\alpha} \partial_{\xi}^{\beta} q(s, x, \xi)\right| \leq C(1+|\xi|)^{\Re \mu(s)-|\beta|},
$$

where $\Re \mu(s)$ is the real part of $\mu(s)$ and $C$, which depends on $\alpha, \beta$, is uniform for $x$ and $s$ in compact sets. This implies that the homogeneous terms $q_{j}(s, x, \xi)$ in the symbol expansion of $Q_{s}$ are analytic in $s$, that the kernel defining $Q_{s}$ away from the diagonal is analytic in $s$, and that if $A: C^{\infty}(E) \rightarrow C^{\infty}(E)$ 
is a positive elliptic second order differential operator, then $Q_{s} A^{-\mu(s) / 2}$ is a analytic family of bounded operators on $L^{2}(E)$.

\section{Variation formulas.}

Let $g$ be a metric on $M$, let $h \in C^{\infty}\left(S^{2} M\right)$, and set

$$
\tilde{g}(\sigma)=g+\sigma h
$$

We use primes to denote differentiation with respect to $\sigma$, so for example

$$
F^{\prime}=\frac{d}{d \sigma} F_{\tilde{g}(\sigma)}, \quad \quad F^{\prime \prime}=\frac{d^{2}}{d \sigma^{2}} F_{\tilde{g}(\sigma)}
$$

In this section we will prove the following Lemma expressing the second variation of $Z(s)$ in terms of $F, F^{\prime}$ and $F^{\prime \prime}$.

\section{Lemma 3.1.}

$$
\begin{aligned}
& \frac{d^{2} \mathcal{Z}(s)}{d \sigma^{2}}=\operatorname{trace} F^{\prime \prime} L(s)-\frac{2}{\Gamma(s-n / 2)} \operatorname{trace}\left(F^{\prime} \Pi F^{\prime} F^{-1} \int_{1}^{\infty} t^{s}\left(e^{-t F}-\Pi\right) d t\right) \\
& +\frac{1}{\Gamma(s-n / 2)} \operatorname{trace} \iint_{u+v>1}(u+v)^{s} F^{\prime}\left(e^{-u F}-\Pi\right) F^{\prime}\left(e^{-v F}-\Pi\right) d u d v \\
& \quad+\frac{1}{\Gamma(s-n / 2)} \operatorname{trace} \iint_{u+v<1}(u+v)^{s} F^{\prime} e^{-u F} F^{\prime} e^{-v F} d u d v .
\end{aligned}
$$

We start by expressing the first variation in terms of $F$ and $F^{\prime}$. In the case when the kernel of $F$ is trivial,

$$
\mathcal{Z}(s)=\frac{\Gamma(s)}{\Gamma(s-n / 2)} \operatorname{trace} F^{-s}
$$

and

$$
D \mathcal{Z}(s)(h)=\frac{d}{d \sigma} \mathcal{Z}(s)=\frac{-\Gamma(s+1)}{\Gamma(s-n / 2)} \operatorname{trace} F^{\prime} F^{-s-1} .
$$

When $F$ has a non-trivial kernel whose dimension does not vary with $\sigma$, let $\Pi$ denote the projection onto the kernel, and write

$$
F^{-s}=(F+\Pi)^{-s}-\Pi,
$$

so (3.2) still holds. Using $\Pi^{2}=\Pi$ we get

$$
\Pi^{\prime} \Pi+\Pi \Pi^{\prime}=\Pi^{\prime}
$$

and using $F \Pi=0$ we get

$$
F^{\prime} \Pi=-F \Pi^{\prime}
$$


$\mathrm{SO}$

$$
\Pi F^{\prime} \Pi=0
$$

and

$$
\text { trace } F^{\prime} \Pi=0 \text {. }
$$

Hence

$$
\operatorname{trace} \Pi^{\prime}(F+\Pi)^{-s-1}=2 \operatorname{trace} \Pi^{\prime} \Pi(F+\Pi)^{-s-1}=2 \operatorname{trace} \Pi^{\prime} \Pi=\operatorname{trace} \Pi^{\prime}=0
$$

and

$$
\begin{aligned}
D \mathcal{Z}(s)(h)=\frac{-\Gamma(s+1)}{\Gamma(s-n / 2)} \operatorname{trace}(F+\Pi)^{\prime}(F+\Pi)^{-s-1}=\frac{-\Gamma(s+1)}{\Gamma(s-n / 2)} & \operatorname{trace} F^{\prime}(F+\Pi)^{-s-1} \\
= & \frac{-\Gamma(s+1)}{\Gamma(s-n / 2)} \operatorname{trace} F^{\prime} F^{-s-1} .
\end{aligned}
$$

It will be convenient to write this in the form

$$
D Z(s)(h)=\operatorname{trace} F^{\prime} L(s)
$$

where

$$
L(s)=\frac{-1}{\Gamma(s-n / 2)}\left(\Gamma(s+1) F^{-s-1}+\frac{1}{s+1} \Pi\right) .
$$

Now we compute the second variation of $\mathcal{Z}(s)$ in terms of $F, F^{\prime}$, and $F^{\prime \prime}$. In the case when the kernel of $F$ is trivial,

$$
\frac{d^{2} \mathcal{Z}(s)}{d \sigma^{2}}=\frac{-\Gamma(s+1)}{\Gamma(s-n / 2)} \operatorname{trace} F^{\prime \prime} F^{-s-1}-\frac{\Gamma(s+1)}{\Gamma(s-n / 2)} \operatorname{trace} F^{\prime} \frac{d \tilde{F}^{-s-1}}{d \sigma} .
$$

Using the Melin transform, we write

$$
\tilde{F}^{-s-1}=\frac{1}{\Gamma(s+1)} \int_{0}^{\infty} t^{s} e^{-t \tilde{F}} d t
$$

By Duhamel's principle,

$$
\frac{\partial e^{-t \tilde{F}}}{\partial \sigma}=-\int_{u=0}^{t} e^{-u \tilde{F}} \frac{d \tilde{F}}{d \sigma} e^{-(t-u) \tilde{F}} d u
$$

and so

$$
\begin{aligned}
\left.\frac{\partial \tilde{F}^{-s-1}}{\partial \sigma}\right|_{\sigma=0} & =\left.\frac{1}{\Gamma(s+1)} \int_{0}^{\infty} t^{s} \frac{\partial e^{-t \tilde{F}}}{\partial \sigma}\right|_{\sigma=0} d t=-\frac{1}{\Gamma(s+1)} \int_{0}^{\infty} \int_{0}^{t} t^{s} e^{-u F} F^{\prime} e^{-(t-u) F} d u d t \\
= & -\frac{1}{\Gamma(s+1)} \int_{0}^{\infty} \int_{0}^{\infty}(u+v)^{s} e^{-u F} F^{\prime} e^{-v F} d u d v .
\end{aligned}
$$


Hence

$$
\begin{aligned}
& \frac{d^{2} \mathcal{Z}(s)}{d \sigma^{2}} \\
& \quad=\frac{-\Gamma(s+1)}{\Gamma(s-n / 2)} \operatorname{trace} F^{\prime \prime} F^{-s-1}+\frac{1}{\Gamma(s-n / 2)} \operatorname{trace} \int_{u=0}^{\infty} \int_{v=0}^{\infty}(u+v)^{s} F^{\prime} e^{-u F} F^{\prime} e^{-v F} d u d v .
\end{aligned}
$$

In the case when $F$ has non trivial smoothly varying kernel, we have $\Pi F=0=F \Pi$, so we get the relations

$$
F \Pi^{\prime}=-F^{\prime} \Pi, \quad \Pi^{\prime} F=-\Pi F^{\prime} .
$$

They imply in particular that

$$
F \Pi^{\prime} F=0, \quad \Pi F^{\prime} \Pi=0 .
$$

Since $\Pi^{\prime}=\Pi^{\prime} \Pi+\Pi \Pi^{\prime}$, we also have

$$
\Pi \Pi^{\prime} \Pi=0,
$$

so

$$
(F+\Pi) \Pi^{\prime}(F+\Pi)=F \Pi^{\prime} \Pi+\Pi \Pi^{\prime} F=-F^{\prime} \Pi-\Pi F^{\prime} .
$$

Hence

$$
\Pi^{\prime}=-(F+\Pi)^{-1} F^{\prime} \Pi-\Pi F^{\prime}(F+\Pi)^{-1}=-F^{-1} F^{\prime} \Pi-\Pi F^{\prime} F^{-1} .
$$

We define

$$
\Psi_{s}(\mu)= \begin{cases}\left(1-\mu^{-s-1}\right) /(\mu-1) & \mu \neq 0,1 \\ s+1 & \mu=1 \\ 0 & \mu=0\end{cases}
$$

Then when $\mu>0$,

$$
\int_{0}^{\infty} \int_{0}^{\infty}(u+v)^{s} e^{-u} e^{-v \mu} d u d v=\Gamma(s+1) \Psi_{s}(\mu) .
$$

Starting from the formula

$$
\frac{d \mathcal{Z}(s)}{d \sigma}=\frac{-\Gamma(s+1)}{\Gamma(s-n / 2)} \operatorname{trace} F^{\prime}(F+\Pi)^{-s-1}
$$

and following the previous argument gives

$$
\begin{aligned}
\frac{d^{2} \mathcal{Z}(s)}{d \sigma^{2}}= & \frac{-\Gamma(s+1)}{\Gamma(s-n / 2)} \operatorname{trace} F^{\prime \prime}(F+\Pi)^{-s-1} \\
& \quad+\frac{1}{\Gamma(s-n / 2)} \operatorname{trace} \int_{0}^{\infty} \int_{0}^{\infty}(u+v)^{s} F^{\prime} e^{-u(F+\Pi)}(F+\Pi)^{\prime} e^{-v(F+\Pi)} d u d v .
\end{aligned}
$$

Furthermore,

$$
\begin{aligned}
\operatorname{trace} \int_{0}^{\infty} \int_{0}^{\infty}(u+v)^{s} F^{\prime} e^{-u(F+\Pi)} \Pi^{\prime} e^{-v(F+\Pi)} d u d v & \\
= & -2 \operatorname{trace} \int_{0}^{\infty} \int_{0}^{\infty}(u+v)^{s} F^{\prime} e^{-u(F+\Pi)} \Pi F^{\prime} F^{-1} e^{-v(F+\Pi)} d u d v \\
= & -2 \operatorname{trace}\left(F^{\prime} \Pi F^{\prime} F^{-1} \int_{0}^{\infty} \int_{0}^{\infty}(u+v)^{s} e^{-u} e^{-v(F+\Pi)} d u d v\right) \\
& =-2 \Gamma(s+1) \text { trace } \Pi F^{\prime} F^{-1} \Psi_{s}(F) F^{\prime},
\end{aligned}
$$


and since

$$
e^{-u(F+\Pi)}=e^{-u F}-\Pi+e^{-u} \Pi,
$$

we get

$$
\begin{aligned}
F^{\prime} e^{-u(F+\Pi)} F^{\prime} e^{-v(F+\Pi)=} F^{\prime}\left(e^{-u F}-\Pi\right) F^{\prime}\left(e^{-v F}-\Pi\right) & \\
& +e^{-u} F^{\prime} \Pi F^{\prime} e^{-v F}+e^{-v} F^{\prime} e^{-u F} F^{\prime} \Pi,
\end{aligned}
$$

and

$$
\begin{aligned}
\operatorname{trace} \int_{0}^{\infty} \int_{0}^{\infty}(u+v)^{s} F^{\prime} e^{-u(F+\Pi)} F^{\prime} e^{-v(F+\Pi)} d u d v & \\
=\operatorname{trace} \int_{0}^{\infty} \int_{0}^{\infty}(u+v)^{s} F^{\prime}\left(e^{-u F}-\Pi\right) F^{\prime} & \left(e^{-v F}-\Pi\right) d u d v \\
& +2 \Gamma(s+1) \operatorname{trace} \Pi F^{\prime} \Psi_{s}(F) F^{\prime} .
\end{aligned}
$$

Moreover

$$
\operatorname{trace} F^{\prime \prime} \Pi=\operatorname{trace}\left(F^{\prime} \Pi\right)^{\prime}-\operatorname{trace} F^{\prime} \Pi^{\prime}=-\operatorname{trace} F^{\prime} \Pi^{\prime}=2 \operatorname{trace} \Pi F^{\prime} F^{-1} F^{\prime}
$$

and

2 trace $\Pi F^{\prime} \Psi_{s}(F) F^{\prime}-2$ trace $\Pi F^{\prime} F^{-1} \Psi_{s}(F) F^{\prime}-2$ trace $\Pi F^{\prime} F^{-1} F^{\prime}=-2$ trace $\Pi F^{\prime} F^{-s-2} F^{\prime}$.

Hence (3.5) becomes

$$
\begin{aligned}
\frac{d^{2} \mathcal{Z}(s)}{d \sigma^{2}}=\frac{-\Gamma(s+1)}{\Gamma(s-n / 2)} & \operatorname{trace} F^{\prime \prime} F^{-s-1}-\frac{2 \Gamma(s+1)}{\Gamma(s-n / 2)} \operatorname{trace} \Pi F^{\prime} F^{-s-2} F^{\prime} \\
& +\frac{1}{\Gamma(s-n / 2)} \operatorname{trace} \int_{0}^{\infty} \int_{0}^{\infty}(u+v)^{s} F^{\prime}\left(e^{-u F}-\Pi\right) F^{\prime}\left(e^{-v F}-\Pi\right) d u d v .
\end{aligned}
$$

We will reorganize this formula to combine terms which contain poles. We have

$$
\begin{aligned}
& \operatorname{trace} \iint_{u+v<1}(u+v)^{s} F^{\prime}\left(e^{-u F}-\Pi\right) F^{\prime}\left(e^{-v F}-\Pi\right) d u d v= \\
& \operatorname{trace} \iint_{u+v<1}(u+v)^{s} F^{\prime} e^{-u F} F^{\prime} e^{-v F} d u d v-2 \operatorname{trace} \iint_{u+v<1}(u+v)^{s} F^{\prime} \Pi F^{\prime}\left(e^{-v F}-\Pi\right) d u d v \\
& \quad=\operatorname{trace} \iint_{u+v<1}(u+v)^{s} F^{\prime} e^{-u F} F^{\prime} e^{-v F} d u d v+2 \operatorname{trace} F^{\prime} \Pi F^{\prime} F^{-1}\left(\int_{0}^{1} t^{s} e^{-t F} d t-\frac{I}{s+1}\right) .
\end{aligned}
$$

So defining $L(s)$ as in (3.4), we obtain Lemma 3.1.

\section{Reducing to the heat kernel at small times.}

Our aim in this paper is to express the Hessian of $Z(s)$ in the form given in (1.4). In this Section we reduce to a local problem involving the heat kernel at small times. Most of the steps in this reduction are quite routine. 
We start by expressing $F$ defined in (1.2) in local coordinates. Let $S_{+}^{2}\left(\mathbb{R}^{n}\right)$ be the cone of positive definite elements of $S^{2}\left(\mathbb{R}^{n}\right)$. There exist functions $a, b, c$, such that in the natural trivialization of $E$ associated to arbitrary local coordinates on $M$,

$$
F=I V^{2 / n} \sum_{i j} g^{i j} \partial_{i} \partial_{j}+\sum_{i} a^{i} \partial_{i}+b+\sum_{i, j, k, \ell}\left(\partial_{k} \partial_{\ell} g^{i j}\right) c_{i j}^{k \ell}
$$

where $I$ is the identity $N \times N$ matrix, and

$$
\begin{array}{llr}
a:(0, \infty) \times S_{+}^{2}\left(\mathbb{R}^{n}\right) \times T^{0,3}\left(\mathbb{R}^{n}\right) & \rightarrow & T^{1,0}\left(\mathbb{R}^{n}\right) \otimes T^{1,1}\left(\mathbb{R}^{N}\right), \\
b:(0, \infty) \times S_{+}^{2}\left(\mathbb{R}^{n}\right) \times T^{0,3}\left(\mathbb{R}^{n}\right) & \rightarrow & T^{1,1}\left(\mathbb{R}^{N}\right), \\
c:(0, \infty) \times S_{+}^{2}\left(\mathbb{R}^{n}\right) & \rightarrow & T^{2,2}\left(\mathbb{R}^{n}\right) \otimes T^{1,1}\left(\mathbb{R}^{N}\right)
\end{array}
$$

are smooth functions which are independent of the metric and the choice of local coordinates, so writing $\partial g$ for the tensor $\left(\partial_{i} g_{j k}\right) \in T^{0,3}\left(\mathbb{R}^{n}\right)$ at each point of the coordinate chart,

$$
\begin{array}{cl}
\text { for } \quad 1 \leq i \leq n, & a^{i}=a^{i}(V, g, \partial g) \in T^{1,1}\left(\mathbb{R}^{N}\right), \\
& b=b(V, g, \partial g) \in T^{1,1}\left(\mathbb{R}^{N}\right), \\
\text { for } \quad 1 \leq i, j, k, \ell \leq n, & c_{i j}^{k \ell}=c_{i j}^{k \ell}(V, g) \in T^{1,1}\left(\mathbb{R}^{N}\right) .
\end{array}
$$
by

In the previous Section we computed the second variation of $Z(s)$ when the varying metric is given

$$
\tilde{g}(\sigma)=g+\sigma h
$$

Differentiating (4.1) once, we obtain

$$
F^{\prime}=\sum_{\substack{i, j \\|\alpha|+|\beta| \leq 2}}\left(\partial^{\alpha} h_{i j}\right) A_{\alpha \beta}^{i j} \partial^{\beta}, \quad A_{\alpha \beta}^{i j}=A_{\alpha \beta}^{i j}\left(V, \partial^{\gamma} g\right) \in T^{1,1}\left(\mathbb{R}^{N}\right),
$$

where $\gamma$ ranges over multiindices with $|\beta|+|\gamma| \leq 2$. Differentiating (4.1) twice we obtain

$$
F^{\prime \prime}=\sum_{\substack{i, j, k, \ell \\|\alpha|+|\beta| \leq 2,|\gamma| \leq 2}}\left(\partial^{\alpha} h_{k \ell}\right)\left(\partial^{\beta} h_{i j}\right) B_{\alpha \beta \gamma}^{i j k \ell} \partial^{\gamma}, \quad B_{\alpha \beta \gamma}^{i j k \ell}=B_{\alpha \beta \gamma}^{i j k \ell}\left(V, \partial^{\delta} g\right) \in T^{1,1}\left(\mathbb{R}^{N}\right) .
$$

The Hessian of $Z(s)$ with respect to the flat connection on the space of metrics is just given by

$$
\operatorname{Hess} Z(s)(h, h)=\frac{d^{2}}{d \sigma^{2}} Z(s)
$$

We will show that if we can prove Theorem 1 in this case, then the result also follows when we use the intrinsic connection on the space of metrics. We also reduce (1.4) to the case when $\langle\langle g, h\rangle\rangle_{g}=0$. We will then express the second variation given in Lemma 3.1, in terms of $h$, and analyze the terms which arise. 
Definition. Write $\pi_{j}: M \times M \rightarrow M$ for the projection onto the $j^{\text {th }}$ factor. Fix a metric $g$ on $M$. For a pseudodifferential operator $Q: C^{\infty}(E) \rightarrow C^{\infty}(E)$ of order less than $-n$, define $K(Q, x, y)$ to be the continuous section of $\pi_{1}^{*} E \otimes \pi_{2}^{*} E^{*}$ on $M \times M$ such that $K(Q, x, y) d V(y)$ is the integral kernel of $Q$. If $Q(s)$ is an analytic family of polyhomogeneous pseudodifferential operators then $K(Q(s), x, y)$ may be analytically continued from the $s$-region where $Q(s)$ has order less than $-n$, and we write $K(Q(s), x, y)$ to denote this analytic continuation. When $x \neq y$, the section $K(Q(s), x, y)$ is entire in $s$ and $K(Q(s), x, y) d V(y)$ gives the Schwartz kernel of $Q(s)$ away from the diagonal. At the diagonal, $K(Q(s), x, x)$ is a meromrphic in $s$. To compute the poles and the trivial zeros of $K(Q(s), x, x)$, we refer the reader to [Ok1] Sections 2 and 3.

From (3.3) and (4.2),

$$
\begin{aligned}
D \mathcal{Z}(s)(h)=\operatorname{trace} F^{\prime} L(s)=\int_{M} \operatorname{trace} K\left(F^{\prime} L(s), x, x\right) d V(x) & \\
= & \sum_{\substack{i, j \\
|\alpha|+|\beta| \leq 2}} \int\left(\partial^{\alpha} h_{i j}(x)\right) K\left(A_{\alpha \beta}^{i j} \partial^{\beta} L(s), x, x\right) d V(x) .
\end{aligned}
$$

By introducing a partition of unity and integrating by parts, we can express this in the form

$$
\langle\langle h, X(s)\rangle\rangle_{g}
$$

where $X(s) \in C^{\infty}\left(S^{2} M\right)$. The expression for $L(s)$ in (3.4) was chosen precisely so that (3.3) holds and

$$
K\left(A_{\alpha \gamma}^{i j} \partial^{\alpha} L(s), x, x\right)
$$

is an entire function of $s$.

Now we reduce Theorem 1 to the case when the following two conditions hold:

(a). We take the flat connection on $\mathcal{M}$

(b). $\langle\langle h, g\rangle\rangle_{g}=0$.

To make this reduction, assume that (1.4) holds when (a) and (b) are satisfied. We will first remove condition (b). For general $h \in C^{\infty}\left(S^{2}(T M)\right)$, set

$$
\tilde{\tilde{g}}(\sigma, \tau)=e^{\tau}(g+\sigma h)
$$

so

$$
\partial_{\sigma} \tilde{\tilde{g}}(0,0)=h, \quad \partial_{\tau} \tilde{\tilde{g}}(0,0)=g .
$$

Then $\mathcal{Z}(\tilde{\tilde{g}})$ is independent of $\tau$, so from (2.1) we see that

$$
\operatorname{Hess}_{\text {flat }} \mathcal{Z}(s)(h, g)=-D Z(s)\left(\partial_{\sigma} \partial_{\tau} \tilde{g}\right)=-D \mathcal{Z}(s)(h) .
$$

Define $P: C^{\infty}\left(S^{2} M\right) \rightarrow C^{\infty}\left(S^{2} M\right)$ by

$$
P h=h-c_{h} g, \quad \text { where } \quad c_{h}=\frac{1}{n V}\langle\langle h, g\rangle\rangle_{g},
$$


Then defining $X(s)$ as in (4.4),

$$
\begin{aligned}
& \operatorname{Hess}_{\text {flat }} \mathcal{Z}(s)(h, h)=\operatorname{Hess}_{\text {flat }} \mathcal{Z}(s)(P h, P h)+2 c_{h} \operatorname{Hess}_{\text {flat }} \mathcal{Z}(s)(h, g)+c_{h}^{2} \operatorname{Hess}_{\text {flat }} \mathcal{Z}(s)(g, g) \\
& =\left\langle\left\langle P h, T_{s} P h\right\rangle\right\rangle_{g}-2 c_{h} D \mathcal{Z}(s)(h) \\
& =\left\langle\left\langle P h, T_{s} P h\right\rangle\right\rangle_{g}-\frac{2}{n V}\langle\langle h, g\rangle\rangle_{g}\langle\langle h, X(s)\rangle\rangle_{g} \\
& =\left\langle\left\langle h, P T_{s} P h\right\rangle\right\rangle_{g}-\left\langle\left\langle h, \frac{2}{n V}\langle\langle g, h\rangle\rangle X(s)\right\rangle\right\rangle_{g}
\end{aligned}
$$

The operator corresponding to the first term is $P T_{s} P$ which is a $\Psi D O$ equal to $T_{s}$ up to the smoothing operator $T_{s}-P T_{s} P$. The operator corresponding to the second term is $h \rightarrow \frac{2}{n V}\langle\langle g, h\rangle\rangle X(s)$ which is smoothing and analytic in $s$. This removes condition (b).

By (2.2), for the intrinsic connection on $\mathcal{M}$ we have

$$
\operatorname{Hess}_{\text {intrinsic }} \mathcal{Z}(s)(h, h)=\operatorname{Hess}_{\text {flat }} \mathcal{Z}(s)(h, h)+D \mathcal{Z}(s)(w)
$$

where

$$
w_{i j}=-\sum_{k, \ell} h_{i k} g^{k \ell} h_{\ell j}+\frac{1}{2}\langle h, g\rangle_{g} h_{i j}-\frac{1}{4}\langle h, h\rangle_{g} g_{i j}
$$

Then raising indices with respect to the metric $g$ in the usual way,

$$
D \mathcal{Z}(s)(w)=-\int \sum_{i, j, k, \ell} h_{i k} g^{k \ell} h_{\ell j} X^{i j}(s) d V+\frac{1}{2} \int_{M}\langle h, g\rangle_{g}\langle h, X(s)\rangle_{g} d V-\frac{1}{4} \int_{M}\langle h, h\rangle_{g}\langle g, X(s)\rangle_{g} d V
$$

The operator corresponding to this form is a degree zero operator which is analytic in $s$.

This reduces Theorem 1 to the case when we take the flat connection on $\mathcal{M}$ and assume that $h$ is trace free relative to $g$. In particular we now have $\tilde{V}^{\prime}(0)=0$. The rest of this section is devoted to analyzing the terms of the Hessian of $\mathcal{Z}(s)$ in Lemma 3.1, on the right hand side of (3.1).

With $L(s)$ defined in (3.4), from (4.3) we have

$$
\operatorname{trace} F^{\prime \prime} L(s)=\int_{M} \sum_{\substack{i, j, k, \ell \\|\alpha|+|\beta| \leq 2,|\gamma| \leq 2}}\left(\partial^{\alpha} h_{k \ell}\right)\left(\partial^{\beta} h_{i j}\right) \operatorname{trace} K\left(B_{\alpha \beta \gamma}^{i j k \ell} \partial^{\gamma} L(s), x, x\right) d V(x)
$$

The operator corresponding to this form has degree 2 and is entire in $s$.

To deal with the remaining three terms in (3.1) we will need the following standard estimates on the asymptotics of the heat kernel $K\left(e^{-t F}, x, y\right)$. Write $d$ for the geodesic distance between points $x$ and $y$, and write $\nabla$ for the connection on $E$ or $E^{*}$ associated to $g$. We take the norms $|\cdot|_{g}$ associated to $g$ on the fibers $T_{(x, y)}^{p, q}(M \times M)$. There exists $\varepsilon>0$ such that for all $j, k, \ell$,

$$
\begin{array}{ll}
\sup _{d(x, y)>d_{0}}\left|\partial_{t}^{j} \nabla_{x}^{k} \nabla_{y}^{\ell} K\left(e^{-t F}, x, y\right)\right| \leq C e^{-\varepsilon / t}, & \text { when } t<1, \\
\sup _{x, y \in M}\left|\partial_{t}^{j} \nabla_{x}^{k} \nabla_{y}^{\ell} K\left(e^{-t F}-\Pi, x, y\right)\right| \leq C e^{-\varepsilon t}, & \text { when } t>1,
\end{array}
$$


where the constant $C$ depends on $j, k, \ell$. Moreover, if we write $\pi_{i}:[0,1] \times M \times M \rightarrow M, i=1,2$ for the projection onto the $i$ th $M$ factor, and $d(x, y)$ for the distance from $x$ to $y$, there exists $b \in$ $C^{\infty}\left(\pi_{1}^{*} E \otimes \pi_{2}^{*} E^{*}\right)$ and $d_{0}>0, \varepsilon>0$ such that

$$
K\left(e^{-t F}, x, y\right)=\frac{1}{(4 \pi t)^{n / 2} V} e^{-d^{2} /\left(4 t V^{2 / n}\right)} b(t, x, y), \quad \text { when } t<1, d<d_{0} .
$$

Moreover, $b(0, x, x)$ is the identity matrix in $E_{x} \otimes E_{x}^{*}$. See for example [Ch].

By (4.7), we see that

$$
\int_{1}^{\infty} t^{s} K\left(e^{-t F}-\Pi, x, y\right) d t
$$

is a smooth kernel which is entire in $s$, and so there exists a smoothing operator $P(s)$ which is entire in $s$ such that

$$
\operatorname{trace}\left(F^{\prime} \Pi F^{\prime} F^{-1} \int_{1}^{\infty} t^{s}\left(e^{-t F}-\Pi\right) d t\right)=\langle\langle h, P(s) h\rangle\rangle
$$

Now consider the term from (3.1):

$$
\begin{aligned}
& \operatorname{trace} \iint_{u+v>1}(u+v)^{s} F^{\prime}\left(e^{-u F}-\Pi\right) F^{\prime}\left(e^{-v F}-\Pi\right) d u d v \\
& =\operatorname{trace} \int_{1}^{\infty} \int_{1}^{\infty} \quad " \quad d u d v \\
& +\operatorname{trace} \int_{u=0}^{1 / 2} \int_{v=1-u}^{\infty} \quad " \quad d u d v \\
& +\operatorname{trace} \int_{v=0}^{1 / 2} \int_{u=1-v}^{\infty} \quad " \quad d u d v \\
& =(\mathrm{I})+(\mathrm{II})+(\mathrm{III}) \text {. }
\end{aligned}
$$

In any of these terms we can pass the trace inside the integral. Indeed, writing $\|\cdot\|$ for the operator norm, $\|\cdot\|_{1}$ for the trace class norm and and $\|\cdot\|_{2}$ for the Hilbert-Schmidt norm of an operator, when $u>1 / 2$,

$$
\begin{array}{r}
\left\|F^{\prime}\left(e^{-u F}-\Pi\right) F^{\prime}\left(e^{-v F}-\Pi\right)\right\|_{1} \leq\left\|F^{\prime}\left(e^{-u F / 2}-\Pi\right)\right\|_{2}\left\|\left(e^{-u F / 2}-\Pi\right) F^{\prime}\right\|_{2}\left\|\left(e^{-v F}-\Pi\right)\right\| \\
\leq C e^{-\varepsilon u},
\end{array}
$$

where the last inequality follows from (4.7). This shows for example that

$$
\operatorname{trace} \int_{v=0}^{1 / 2} \int_{u=1-v}^{\infty}(u+v)^{s}\left\|F^{\prime}\left(e^{-u F}-\Pi\right) F^{\prime}\left(e^{-v F}-\Pi\right)\right\|_{1} d u d v<\infty
$$

so that the trace can be passed through the integral in (III).

Now we localize by writing $1=\sum_{\chi \in \Lambda} \chi$ where $\Lambda$ is a finite partition of unity on $M$ such that for each point $x \in M$, all the functions $\chi \in \Lambda$ which contain $x$ in their supports are supported in a single 
contractible coordinate chart, $\Omega$ over which $E$ is trivial, and on which $\partial^{\alpha} g_{i j}$, and $\partial^{\alpha} g^{i j}$ are uniformly bounded for each $\alpha$. Hence we express

$$
\begin{aligned}
& \operatorname{trace} F^{\prime}\left(e^{-u F}-\Pi\right) F^{\prime}\left(e^{-v F}-\Pi\right) \\
& =\sum_{\substack{\chi, \tilde{\chi} \in \Lambda, i, j, k, \ell \\
|\alpha|+|\beta| \leq 2,|\gamma|+|\delta| \leq 2}} \operatorname{trace} \chi A_{\alpha \beta}^{i j}\left(\partial^{\alpha} h_{i j}\right) \partial^{\beta}\left(e^{-u F}-\Pi\right) \tilde{\chi} A_{\gamma \delta}^{k \ell}\left(\partial^{\gamma} h_{k \ell}\right) \partial^{\delta}\left(e^{-v F}-\Pi\right) .
\end{aligned}
$$

We can analyze each term in the above sum separately. Fix $\chi, \tilde{\chi}, i, j, k, \ell, \alpha, \beta, \gamma, \delta$ and set (4.12) $\tilde{E}(u, v, x, y)=\chi(x) \tilde{\chi}(y)$ trace $A_{\alpha \beta}^{i j}(x)\left(\partial_{x}^{\beta} K\left(e^{-u F}-\Pi, x, y\right)\right) A_{\gamma \delta}^{k \ell}(y)\left(\partial_{y}^{\delta} K\left(e^{-v F}-\Pi, y, x\right)\right)$. The trace on the right is the trace on $N \times N$ matrices. Then

$$
\begin{aligned}
& \operatorname{trace} \chi A_{\alpha \beta}^{i j}\left(\partial^{\alpha} h_{i j}\right) \partial^{\beta}\left(e^{-u F}-\Pi\right) \tilde{\chi} A_{\gamma \delta}^{k \ell}\left(\partial^{\gamma} h_{k \ell}\right) \partial^{\delta}\left(e^{-v F}-\Pi\right) \\
& =\iint_{\operatorname{Supp}(\chi) \times \operatorname{Supp}(\tilde{\chi})}\left(\partial^{\alpha} h_{i j}(x)\right) \tilde{E}(u, v, x, y)\left(\partial^{\gamma} h_{k \ell}(y)\right) \sqrt{g(x)} \sqrt{g(y)} d x d y .
\end{aligned}
$$

Now from (4.7), for $u>1$ and $v>1$ and each $\alpha, \beta$,

$$
\left|\partial_{x}^{\alpha} \partial_{y}^{\beta} \tilde{E}(u, v, x, y)\right| \leq C e^{-\varepsilon(u+v)} .
$$

Hence

$$
K_{s}^{(I)}(x, y):=\frac{1}{\Gamma(s-n / 2)} \int_{1}^{\infty} \int_{1}^{\infty}(u+v)^{s} \tilde{E}(u, v, x, y) d u d v
$$

is smooth in $(s, x, y)$ and entire in $s$ and $(\mathrm{I})$ is a finite sum of terms of the form

$$
\iint_{\operatorname{Supp}(A) \times \operatorname{Supp}(B)}\left(\partial^{\alpha} h_{i j}(x)\right) K_{s}^{(I)}(x, y)\left(\partial^{\gamma} h_{k \ell}(y)\right) \sqrt{g(x)} \sqrt{g(y)} d x d y .
$$

Integrating by parts to take the derivatives from $h$, we find that the kernel of this bilinear form is smooth and entire in $s$. Similarly, write (II) formally as a sum of terms of the form

$$
\iint_{\Omega \times \Omega}\left(\partial^{\alpha} h_{i j}(x)\right)\left(\chi(x) \text { trace } K_{s}^{(I I)}(x, y) \tilde{\chi}(y)\right)\left(\partial^{\gamma} h_{k \ell}(y)\right) \sqrt{g(x)} \sqrt{g(y)} d x d y,
$$

where $K_{s}^{(I I)}(x, y)$ is given by

$$
K_{s}^{(I I)}(x, y)=\int_{u=0}^{1 / 2} \int_{v=1-u}^{\infty}(u+v)^{s} A_{\alpha \beta}^{i j}(x)\left(\partial_{x}^{\beta} K\left(e^{-u F}, x, y\right)\right) A_{\gamma \delta}^{k \ell}(y)\left(\partial_{y}^{\delta} K\left(e^{-v F}, y, x\right)\right) d u d v .
$$

In doing this we are switching the $d x d y$ integration with the $d u d v$ integration, which must properly interpreted and justified, since the integral in (4.16) may be divergent due to $K_{s}^{(I I)}(x, y)$ being singular on the diagonal. The way to proceed is to work in the class of pseudodifferential operators of order $|\beta|$. Define

so

$$
J_{s}^{(I I)}(u, x, y):=A_{\gamma \delta}^{k \ell}(y) \int_{v=1-u}^{\infty}(u+v)^{s}\left(\partial_{y}^{\delta} K\left(e^{-v F}, y, x\right)\right) d v A_{\alpha \beta}^{i j}(x)
$$

$$
K_{s}^{(I I)}(x, y)=\int_{u=0}^{1 / 2}\left(\partial_{x}^{\beta} K\left(e^{-u F}, x, y\right)\right) J_{s}^{(I I)}(u, x, y) d u .
$$

From (4.7), $J_{s}^{(I I)}(u, x, y)$ is smooth for $(s, u, x, y) \in \mathbb{C} \times\left[0, \frac{1}{2}\right] \times \Omega \times \Omega$ and analytic in $s$. We notice that the integrand on the right hand side of (4.17) is the integral kernel of an operator on $C^{\infty}(\Omega)$ whose order $|\beta|$ symbol norms are bounded uniformly in the parameter $u$. This follows easily from the elementary lemmas below, and shows that the integral in (4.17) converges to the Schwartz kernel of an order $|\beta|$ pseudodifferential operator. 
Lemma 4.1. The operators $e^{-u F}$ are psedodifferential operators of order zero, with symbol norms uniformly bounded in the parameter $u$.

This implies that for $\beta$ fixed, the operators $\partial_{x}^{\beta} e^{-u F}$ are psedodifferential operators of order $|\beta|$ with uniformly bounded symbol norms in the parameter $u$.

Lemma 4.2. If $\mathcal{K}(Q, x, y)$ is the Schwartz kernel of a $\Psi D O, Q$, on $M$ of order $m$, and $J(x, y)$ is smooth. Then the product $\mathcal{K}(Q, x, y) J(x, y)$ is the Schwartz kernel of a $\Psi D O$, which we denote by $Q_{J}$, which also has order $m$. If $Q$ depends smoothly on a parameter $p \in \mathbb{R}^{d}$ then so does $Q_{J}$.

We can conclude that the expression in (II) is equal to $(4.16)$, and $K_{s}^{(I I)}(x, y)$ is the Schwartz kernel of a $\Psi D O$ of order $|\beta|$. The question then arises whether this operator is polyhomogeneous and how one obtains its symbol expansion. For a function $\psi \in C^{\infty}([0,1])$, integration by parts gives

$$
\begin{aligned}
\int_{u=0}^{1 / 2} e^{-u \lambda} \psi(u) d u & =\lambda^{-1} \psi(0)+\lambda^{-2} \psi^{\prime}(0)+\ldots+\lambda^{-M} \psi^{(M-1)}(0) \\
& -\left(\lambda^{-1} \psi(1)+\lambda^{-2} \psi^{\prime}(1)+\ldots+\lambda^{-M} \psi^{(M-1)}(1)\right) e^{-\lambda / 2}+\lambda^{-M} \int_{u=0}^{1 / 2} e^{-u \lambda} \psi^{(M)}(u) d u
\end{aligned}
$$

we see that (4.17) equals

$$
\begin{aligned}
\left.\sum_{m=0}^{M-1}\left(\partial_{x}^{\beta} K\left(F^{-m-1}, x, y\right)\right) \partial_{u}^{m}\right|_{u=0} J_{s}^{(I I)}(u, x, y) & -\left.\sum_{m=0}^{M-1}\left(\partial_{x}^{\beta} K\left(F^{-m-1} e^{-F / 2}, x, y\right)\right) \partial_{u}^{m}\right|_{u=1} J_{s}^{(I I)}(u, x, y) \\
& +\int_{u=0}^{1 / 2}\left(\partial_{x}^{\beta} K\left(F^{-M} e^{-u F}, x, y\right)\right) \partial_{u}^{M} J_{s}^{(I I)}(u, x, y) d u .
\end{aligned}
$$

The term in the second sum

$$
-\left.\left(\partial_{x}^{\beta} K\left(F^{-m-1} e^{-F / 2}, x, y\right)\right) \partial_{u}^{m}\right|_{u=1} J_{s}^{(I I)}(u, x, y)
$$

is smooth in $(s, x, y)$ and analytic in $s$. We need the following refinement of Lemma 4.2:

Lemma 4.3. In fact if the $\Psi D O, Q$, in Lemma 4.2 is polyhomogeneous of degree $\mu$, then so is $Q_{J}$, and if $J=J_{s}$ depends analytically on a parameter $s$, then $Q_{J_{s}}$ is an analytic family of pseudodifferential operators.

With this refinement, we see that the term in (4.18)

$$
\left.\left(\partial_{x}^{\beta} K\left(F^{-m-1}, x, y\right)\right) \partial_{u}^{m}\right|_{u=0} J_{s}^{(I I)}(u, x, y) d V(y)
$$

is the Schwartz kernel of a polyhomogeneous $\Psi D O$ operator of degree $|\beta|-2 m-2$. The last term in (4.18) is the Schwartz kernel of a pseudodifferential operator of order $|\beta|-2 M-2$. We conclude that $K_{s}^{(I I)}(x, y)$ is the Schwartz kernel of a polyhomogeneous $\Psi$ DO of order $|\beta|-2$, which is analytic in $s$. The lemmas are consequences of the standard theory. Indeed for Lemma 4.1, following [Gi] one can express the symbol of the heat operator in terms of the symbol of the resolvant via a contour integral. However, the resolvant $(\lambda-F)^{-1}$ is a pseudodifferential operator, and the zeroth order symbol norms 
of $\lambda(\lambda-F)^{-1}$ are uniformly bounded in $\lambda$. Lemmas 4.2 and 4.3 follow from the relationship between amplitudes and symbols, see $[\mathrm{Tr}]$.

We return to our analysis of (4.10). Term (III) is dealt in the same fashion as Term (II) with the roles of $u$ and $v$ interchanged. Now we want to understand the final term in (3.1) which is given in (1.10):

$$
\frac{1}{\Gamma(s-n / 2)} \operatorname{trace} \iint_{u+v<1}(u+v)^{s} F^{\prime} e^{-u F} F^{\prime} e^{-v F} d u d v
$$

From now on we will refer to the steps listed in Table 1.1.

Step 14: Using estimates based on (4.8), we can take the trace in (4.8) inside the integral because

$$
\left\|F^{\prime} e^{-u F} F^{\prime} e^{-v F}\right\|_{1} \leq\left\|F^{\prime} e^{-u F / 2}\right\|_{2}\left\|e^{-u F / 2} F^{\prime}\right\|_{2}\left\|e^{-v F}\right\| \leq C u^{-n-2} .
$$

Since this is also true with $u$ and $v$ interchanged, we have

$$
\left\|F^{\prime} e^{-u F} F^{\prime} e^{-v F}\right\|_{1} \leq C(u+v)^{-n-2}
$$

and so for $\Re s$ sufficiently large,

$$
\iint_{u+v<1}(u+v)^{s}\left\|F^{\prime} e^{-u F} F^{\prime} e^{-v F}\right\|_{1} d u d v<\infty .
$$

Steps 7, 3: Taking a partition of unity $1=\sum_{\chi \in \Lambda} \chi$ on $M$ as for (4.11), we see that

$$
\operatorname{trace} F^{\prime} e^{-u F} F^{\prime} e^{-v F}=\sum_{\substack{\chi, \tilde{\chi} \in \Lambda, i, j, k, \ell \\|\alpha|+|\beta| \leq 2,|\gamma|+|\delta| \leq 2}} \operatorname{trace} \chi A_{\alpha \beta}^{i j}\left(\partial^{\alpha} h_{i j}\right) \partial^{\beta} e^{-u F} \tilde{\chi} A_{\gamma \delta}^{k \ell}\left(\partial^{\gamma} h_{k \ell}\right) \partial^{\delta} e^{-v F}
$$

Step 1: Fixing $\chi, \tilde{\chi} \in \Lambda, i, j, k, \ell, \alpha, \beta, \gamma, \delta$, we will consider each term in (4.20). Set

$$
E(u, v, x, y)=\chi(x) \tilde{\chi}(y) \text { trace } A_{\alpha \beta}^{i j}(x)\left(\partial_{x}^{\beta} K\left(e^{-u F}, x, y\right)\right) A_{\gamma \delta}^{k \ell}(y)\left(\partial_{y}^{\delta} K\left(e^{-v F}, y, x\right)\right) .
$$

Step 10: Then

$$
\begin{aligned}
& \operatorname{trace} \chi A_{\alpha \beta}^{i j}\left(\partial^{\alpha} h_{i j}\right) \partial^{\beta} e^{-u F} \tilde{\chi} A_{\gamma \delta}^{k \ell}\left(\partial^{\gamma} h_{k \ell}\right) \partial^{\delta} e^{-v F} \\
& \quad=\iint_{\operatorname{Supp}(\chi) \times \operatorname{Supp}(\tilde{\chi})}\left(\partial^{\alpha} h_{i j}(x)\right) E(u, v, x, y)\left(\partial^{\gamma} h_{k \ell}(y)\right) \sqrt{g(x)} \sqrt{g(y)} d x d y .
\end{aligned}
$$

Steps 8, 14: Hence formally, (4.19) is a sum of terms of the form

$$
\iint_{\operatorname{Supp}(\chi) \times \operatorname{Supp}(\tilde{\chi})}\left(\partial^{\alpha} h_{i j}(x)\right) K_{s}(x, y)\left(\partial^{\gamma} h_{k \ell}(y)\right) \sqrt{g(x)} \sqrt{g(y)} d x d y
$$

where

$$
K_{s}(x, y):=\frac{1}{\Gamma(s-n / 2)} \iint_{u+v<1}(u+v)^{s} E(u, v, x, y) d u d v
$$


When the supports of $\chi$ and $\tilde{\chi}$ are disjoint we can use (4.6) and (4.8) to see that (4.19) is a bilinear form on $C^{\infty}\left(S^{2} M\right)$ with a smooth kernel which is analytic in $s$. Hence we have reduced to the case when $\chi$ and $\tilde{\chi}$ are supported on a coordinate chart $\Omega$ whose diameter is less than the injectivity radius of $M$.

\section{The symbol of $U_{s}$.}

Here in Section 5, we describe how to compute the symbol expansion of $U_{s}$, and we carry this out explicitly for $u_{s}$ thus obtaining Theorem 2. To do this we expand on the summary in Section 1. Section 5 can be read independently from Section 6 , which contains the rigorous proof of Theorem 1 , and shows that the algorithm we lay down here is correct. We will see in particular in Section 6, that (4.19) is a sum of terms of the form (4.23). Moreover, we see in Lemmas 6.1 and 6.5 that $K_{s}$ defined in (4.24) has symbol

$$
\tilde{U}_{s}(x, \xi)+\tilde{V}_{s}(x, \xi)
$$

where setting

$$
m=|\beta|+|\delta|,
$$

$\tilde{U}_{s}$ is polyhomogeneous of degree $n-2 s-4+m$, and $\tilde{V}_{s}$ polyhomogneous of degree $m-2$.

Step 3: We are interested in obtaining the symbol expansion of $\tilde{U}_{s}$ at the point $x \in M$, and so we work in coordinates on a small coordinate chart $\Omega$ containing $x$.

Steps 11, 12, 13: To obtain the symbol expansion of $\tilde{U}_{s}$, we make an expansion of $E(u, v, x, y)$. Using $(4.8)$,

$$
\partial_{x}^{\beta} K\left(e^{-u F}, x, y\right)=u^{-n / 2} e^{-d^{2}(x, y) / 4 u} b_{\beta}(u, x, y)
$$

where $u^{|\beta|} b_{\beta}(u, x, y)$ is a smooth $N \times N$ valued function on $[0,1] \times \Omega^{2}$ whose Taylor expansion at $u=0$ gives an expansion of the form

$$
b_{\beta}(u, x, y) \sim \sum_{\ell=-|\beta|}^{\infty} u^{\ell} b_{\beta \ell}(x, y), \quad b_{\beta \ell}(x, y)=O\left(d(x, y)^{\max \{-2 \ell-|\beta|, 0\}}\right) .
$$

Here, $b_{\beta \ell}(x, y)$ is smooth on $\Omega^{2}$. Hence considering (4.21),

$$
E(u, v, x, y)=(u v)^{-n / 2} e^{-d^{2}(x, y)(1 / 4 u+1 / 4 v)} J(u, v, x, y)
$$

where

$$
u^{|\beta|} v^{|\delta|} J(u, v, x, y)
$$

is smooth on $[0,1]^{2} \times \Omega^{2}$. and $J(u, v, x, y)$ has an expansion of the form

$$
J(u, v, x, y) \sim \sum_{a=-|\beta|}^{\infty} \sum_{b=-|\delta|}^{\infty} u^{a} v^{b} J_{a b}(x, y), \quad J_{a b}(x, y)=O\left(d(x, y)^{\max \{-2(a+b)-(|\beta|+|\delta|), 0\}}\right) .
$$

Here, $J_{a b}(x, y)$ is smooth on $\Omega^{2}$. 
Step 4: Fix $x$ and write $w$ for geodesic normal coordinates of $y$ centered at $x$. We make a Taylor expansion of $J_{a b}(x, y)$ about $w=0$ to write

$$
J(u, v, x, y) \sim \sum_{\ell=-m}^{\infty} J_{\ell}(u, v, x, y),
$$

where for constants $J_{a b \mu}(x)$,

$$
J_{\ell}(u, v, x, y)=\sum_{2 a+2 b+|\mu|=\ell} J_{a b \mu} u^{a} v^{b} w^{\mu} .
$$

The sum in (5.5) is clearly finite. Now set

$$
\begin{aligned}
& E_{\ell}(u, v, x, y)=(u v)^{-n / 2} e^{-d^{2}(x, y)(1 / 4 u+1 / 4 v)} J_{\ell}(u, v, x, y), \\
& K_{s, \ell}(x, y)=\frac{1}{\Gamma(s-n / 2)} \iint_{u+v<1}(u+v)^{s} E_{\ell}(u, v, x, y) d u d v .
\end{aligned}
$$

Step 15: The symbol of $E_{\ell}(u, v, x, \xi)$ has the form

$$
U_{s, \ell}(x, \xi)+V_{s, \ell}(x, \xi)
$$

where $U_{s, \ell}(x, \xi)$ is homogeneous of degree $n-2 s-4-\ell$, and $V_{s}(x, \xi)$ is polyhomogeneous of degree $m-2$. To see this, the metric-modified symbol (see 2.4) of $E_{\ell}(u, v, x, y)$ is obtained by taking the Fourier transform in $y$,

$$
\sigma\left(E_{\ell}, u, v, x, \xi\right)=\int e^{i w \cdot \xi} E_{\ell}(u, v, x, y) d w
$$

From the definition of $E_{\ell}$,

$$
\begin{gathered}
\sigma\left(E_{\ell}, u, v, x, \xi\right)=(4 \pi)^{n / 2}(u+v)^{-n / 2} \sum_{2 a+2 b+|\mu|=\ell} J_{a b \mu}(x) u^{a} v^{b}\left(-i \partial_{\xi}\right)^{\mu} e^{-|\xi|^{2} u v /(u+v)} \\
=(u+v)^{-n / 2} e^{-|\xi|^{2} u v /(u+v)} \sum_{\substack{2 a+2 b+|\mu|=\ell \\
\nu \leq \mu}} c_{\mu \nu} J_{a b \mu}(x)(u+v)^{-(|\mu|+|\nu|) / 2}(u v)^{(|\mu|+|\nu|) / 2} u^{a} v^{b} \xi^{\nu} \\
=(u+v)^{-n / 2-\ell} e^{-|\xi|^{2} u v /(u+v)} \sum_{\substack{|\nu| \leq 2 m+\ell \\
2 a+2 b=3 \ell+|\nu|}} \tilde{J}_{a b \nu}(x) u^{a} v^{b} \xi^{\nu},
\end{gathered}
$$

where the constants $c_{\mu \nu}$ and functions $\tilde{J}_{a b \nu}(x)$ are defined appropriately by collecting terms. We will prove in Lemma 6.3 that $\tilde{J}_{a b \nu}(x)$ vanishes if $a$ or $b$ is negative, so

$$
\sigma\left(E_{\ell}, u, v, x, \xi\right)=O\left((u+v)^{-n / 2-\ell}\right)
$$


as $u, v \rightarrow 0$.

Step 14: Hence the symbol of $K_{s, \ell}(x, \xi)$ in (4.24) equals

$$
\frac{1}{\Gamma(s-n / 2)} \sum_{\substack{|\nu| \leq \ell \\ 2 a+2 b=3 \ell+|\nu|}} \tilde{J}_{a b \nu}(x) \xi^{\nu} \iint_{u+v<1}(u+v)^{s-n / 2-\ell} u^{a} v^{b} e^{-|\xi|^{2} u v /(u+v)} d u d v
$$

which converges absolutely for $\Re s$ large.

Step 16: By making the change of variables

$$
T=(u+v)|\xi|^{2}, \quad \tau=\frac{u}{u+v},
$$

we see in Lemmas 6.4 and 6.5 (also (1.19)) that this integral extends analytically and to a sum of symbols of degrees $m-2$ and $n-2 s-4-\ell$. The symbol of degree $n-2 s-4-\ell$ is obtained by extending the integral on the right of $(5.9)$ to $(u, v) \in[0, \infty)^{2}$, and so

$$
\begin{aligned}
U_{s, \ell}(x, \xi)= & \sum_{\substack{|\nu| \leq \ell \\
2 a+2 b=3 \ell+|\nu|}} \tilde{J}_{a b \nu}(x) \xi^{\nu}|\xi|^{n-2 s-4-|\nu|-\ell} \\
& \times \quad \frac{\Gamma(s-n / 2-\ell+a+b+2) \Gamma(n / 2-s+\ell-b-1) \Gamma(n / 2-s+\ell-a-1)}{\Gamma(s-n / 2) \Gamma(n-2 s+2 \ell-a-b-2)} .
\end{aligned}
$$

One might hope to complete the analysis of term (4.19) by studying the properties of the remainder term in the Taylor expansion (5.4). However although the order of $U_{s, \ell}$ decreases as $\ell$ increases, the order of $V_{s, \ell}$ does not. We will need to decompose the kernel $E(u, v, x, y)$ in additional ways which pick out the homogeneous terms in the symbol of $\tilde{V}_{s}$. This is carried out in Section 6 . We emphasize that (5.10) gives the term of degree $n-2 s-4-\ell$ in the symbol of $K_{s}(x, y)$. From $(4.23), u_{s}(x, \xi)$ is the symbol of

$$
\sum_{\substack{\chi, \tilde{\chi} \in \Lambda, i, j, k, \ell \\|\alpha|+|\beta| \leq 2,|\gamma|+|\delta| \leq 2}}(-1)^{|\alpha|+|\gamma|}(\sqrt{g(x)} \sqrt{g(y)})^{-1} \partial_{x}^{\alpha} \partial_{y}^{\gamma}\left(\sqrt{g(x)} \sqrt{g(y)} K_{s}(x, y)\right),
$$

which can be computed from (5.10) using the symbolic calculus. It is also easy to go from the metricmodified symbol to the true symbol using the symbolic calculus if one so desires. See [Tr] for details on the symbolic calculus. One needs to be a little careful because we have been working in normal coordinates based at $x$, and in order to apply the usual symbolic calculus formulas to compose operators, one has use the same coordinate system for the points $x$ and $y$. The necessary change of variables formulas can also be found in $[\mathrm{Tr}]$.

Proof of Theorem 2. We want to compute $u_{s}(x, \xi)$ which is the degree $n-2 s$ term in the symbol of (5.11). (As always, in saying this we must assume $s$ is not a half-integer greater than $n / 2-1$, since at these values the symbol of $\tilde{V}(s)$ also contributes to the degree- $(n-2 s)$ term. $)$ Now $U_{s,-m}(x, \xi)$ is the degree $n-2 s-4+m$ term in the symbol of $K_{s}(x, y)$, which is the principal symbol when $\Re s$ is small. Hence from (5.11),

$$
u_{s}(x, \xi)=\sum_{\substack{\chi, \tilde{\chi} \in \Lambda, i, j, k, \ell \\|\alpha|+|\beta| \leq 2,|\gamma|+|\delta| \leq 2}}(-1)^{|\alpha|}(i \xi)^{\alpha+\gamma} U_{s,-m}(x, \xi) .
$$

We will evaluate 5.12 at a fixed point $x=x_{0}$, and from now on we express $x$ and $y$ in geodesic normal coordinates centered at $x_{0}$. In particular, we assume that the derivatives in (4.21) are computed in these coordinates. 
Lemma 5.1. Let $C(s)$ be defined as in (1.7). Define

$$
G=(4 \pi)^{-n / 2}(u+v)^{-n / 2} e^{-|\xi|^{2} u v /(u+v)},
$$

define the polynomial $P\left(\partial^{\alpha}, t, w\right)$ in the variable $w \in \mathbb{R}^{n}$ by

$$
P\left(\partial^{\alpha}, t, w\right)=e^{|w|^{2} / 4 t} \partial_{w}^{\alpha} e^{-|w|^{2} / 4 t},
$$

define

$$
\sigma\left(\partial^{\alpha}, \partial^{\beta}, \partial^{\gamma}, \partial^{\delta}, u, v, x, \xi\right)=(i \xi)^{\alpha+\gamma} P\left(\partial^{\beta}, u,-i \partial_{\xi}\right) P\left(\partial^{\delta}, v,-i \partial_{\xi}\right) G,
$$

and define

$$
u_{s}\left(\partial^{\alpha}, \partial^{\beta}, \partial^{\gamma}, \partial^{\delta}, x, \xi\right)=\frac{1}{C(s) \Gamma(s-n / 2)} \int_{0}^{\infty} \int_{0}^{\infty}(u+v)^{s} \sigma\left(\partial^{\alpha}, \partial^{\beta}, \partial^{\gamma}, \partial^{\delta}, u, v, x, \xi\right) d u d v
$$

Then

$$
u_{s}\left(x_{0}, \xi\right)=C(s) \sum_{\substack{i, j, k, \ell \\|\alpha|+|\beta|=2,|\gamma|+|\delta|=2}} u_{s}\left(\partial^{\alpha}, \partial^{\beta}, \partial^{\gamma}, \partial^{\delta}, x_{0}, \xi\right) \operatorname{trace} A_{\alpha \beta}^{i j}\left(x_{0}\right) A_{\gamma \delta}^{k \ell}\left(x_{0}\right)
$$

Proof of Lemma 5.1. Recall that each term $K_{s}(x, y)$ in (4.24) depends on the parameters $\chi, \tilde{\chi} \in$ $\Lambda, i, j, k, \ell, \alpha, \beta, \gamma, \delta$, and is given by

$$
K_{s}(x, y):=\frac{1}{\Gamma(s-n / 2)} \int_{0}^{\infty} \int_{0}^{\infty}(u+v)^{s} E(u, v, x, y) d u d v .
$$

where

$$
E(u, v, x, y)=\chi(x) \tilde{\chi}(y) \text { trace } A_{\alpha \beta}^{i j}(x)\left(\partial_{x}^{\beta} K\left(e^{-u F}, x, y\right)\right) A_{\gamma \delta}^{k \ell}(y)\left(\partial_{y}^{\delta} K\left(e^{-v F}, y, x\right)\right) .
$$

The degree $n-2 s-4+m$ term in the symbol of $K_{s}$ is $U_{s,-m}(x, \xi)$, where

$$
U_{s,-m}(x, \xi)=\frac{1}{\Gamma(s-n / 2)} \int_{0}^{\infty} \int_{0}^{\infty}(u+v)^{s} \sigma\left(E_{-m}, u, v, x, \xi\right) d u d v
$$

and $E_{-m}$ which gives the lowest order terms of $E$ is defined in (5.6). We will see that writing $w$ for the geodesic normal coordinates of $y$ about $x=x_{0}$, we have

$$
\begin{aligned}
& E_{-m}\left(u, v, x_{0}, y\right) \\
& \quad=\chi\left(x_{0}\right) \tilde{\chi}\left(x_{0}\right)(4 \pi)^{n / 2}(u v)^{-n / 2}(-1)^{|\beta|}\left(\partial_{w}^{\beta} e^{-|w|^{2} / 4 u}\right)\left(\partial_{w}^{\delta} e^{-|w|^{2} / 4 v}\right) \operatorname{trace} A_{\alpha \beta}^{i j}\left(x_{0}\right) A_{\gamma \delta}^{k \ell}\left(x_{0}\right) .
\end{aligned}
$$

To see this, from (5.1) we must compute the "lowest order" terms in the functions

$$
\left.e^{d^{2}(x, y) / 4 u} \partial_{x}^{\beta}\left(e^{-d^{2}(x, y) / 4 u} b(u, x, y)\right)\right|_{x=x_{0}}, \quad \quad e^{d^{2}\left(x_{0}, y\right) / 4 v} \partial_{y}^{\delta}\left(e^{-d^{2}\left(x_{0}, y\right) / 4 v} b\left(v, x_{0}, y\right)\right),
$$


about $u=v=0$ and $y=x_{0}$, where the orders of the terms $u^{a} w^{\mu}$ and $v^{a} w^{\mu}$ are $2 a+|\mu|$. It is clear that to get the lowest order terms, we can replace $b(u, x, y)$ and $b\left(v, x_{0}, y\right)$ by $b\left(0, x_{0}, x_{0}\right)$ which is the identity matrix. This reduces the problem to computing the lowest order terms in the expansions of the functions

$$
\begin{aligned}
& \left.e^{d^{2}(x, y) / 4 u} \partial_{x}^{\beta} e^{-d^{2}(x, y) / 4 u}\right|_{x=x_{0}}, \\
& e^{d^{2}\left(x_{0}, y\right) / 4 v} \partial_{y}^{\delta} e^{-d^{2}\left(x_{0}, y\right) / 4 v} .
\end{aligned}
$$

Now since the derivatives are expressed in geodesic coordinates about $x_{0},(5.21)$ is precisely

$$
e^{|w|^{2} / 4 v} \partial_{w}^{\delta} e^{-|w|^{2} / 4 v}
$$

which is a linear combination of terms $v^{a} w^{\mu}$ with order $2 a+|\mu|=-|\delta|$. On the other hand (5.20) may involve terms coming from the curvature of the metric. However, these are all of higher order than $-|\beta|$, as one can check by expressing $x$ and $y$ in normal coordinates around $x_{0}$, and expanding

$$
d(x, y)^{2}=|x-y|^{2}+O\left(|x-y|^{3}\right) .
$$

We thus find that the lowest order terms in (5.20) are given by

$$
\left.e^{|x-y|^{2} / 4 u} \partial_{x}^{\beta} e^{-|x-y|^{2} / 4 u}\right|_{x=x_{0}}=(-1)^{|\beta|} e^{|w|^{2} / 4 u} \partial_{w}^{\beta} e^{-|w|^{2} / 4 u}
$$

From this, we get (5.19). Since the Fourier transform of $(4 \pi)^{n / 2}(u v)^{-n / 2} e^{-|y|^{2}(1 / 4 u+1 / 4 v)}$ is $G$, taking the Fourier transform of $E_{-m}$ gives

$$
\sigma\left(E_{-m}, u, v, x_{0}, \xi\right)=\chi\left(x_{0}\right) \tilde{\chi}\left(x_{0}\right) \operatorname{trace} A_{\alpha \beta}^{i j}\left(x_{0}\right) A_{\gamma \delta}^{k \ell}\left(x_{0}\right)(-1)^{|\beta|} P\left(\partial^{\beta}, u,-i \partial_{\xi}\right) P\left(\partial^{\delta}, v,-i \partial_{\xi}\right) G .
$$

Plugging this into (5.12) and (5.18) and summing over $\chi, \tilde{\chi} \in \Lambda$ yields Lemma 5.1.

To complete the proof of Theorem 2 , it remains to compute $u\left(\partial^{\alpha}, \partial^{\beta}, \partial^{\delta}, \partial^{\gamma}, u, v, x, \xi\right)$ for explicit values of $\alpha, \beta, \gamma, \delta$. Now

$$
\begin{array}{ll}
P\left(\partial_{j}, u, w\right)=-\frac{w_{j}}{2 u}, & P\left(\partial_{j}, u,-i \partial_{\xi}\right)=\frac{i}{2 u} \partial_{\xi_{j}} . \\
P\left(\partial_{j} \partial_{k}, u, w\right)=\left(\frac{w_{j} w_{k}}{4 u^{2}}-\frac{\delta_{j k}}{2 u}\right) & P\left(\partial_{j} \partial_{k}, u,-i \partial_{\xi}\right)=-\left(\frac{1}{4 u^{2}} \partial_{\xi_{j}} \partial_{\xi_{k}}+\frac{\delta_{j k}}{2 u}\right),
\end{array}
$$

and

$$
\begin{gathered}
\partial_{\xi_{j}} G=-\frac{2 u v}{u+v} \xi_{j} G, \\
\partial_{\xi_{j}} \partial_{\xi_{k}} G=\left(\frac{4 u^{2} v^{2}}{(u+v)^{2}} \xi_{j} \xi_{k}-\frac{2 u v}{u+v} \delta_{j k}\right) G, \\
\partial_{\xi_{j}} \partial_{\xi_{k}} \partial_{\xi_{p}} G=\left(-\frac{8 u^{3} v^{3}}{(u+v)^{3}} \xi_{j} \xi_{k} \xi_{p}+\frac{4 u^{2} v^{2}}{(u+v)^{2}}\left(\delta_{j k} \xi_{p}+\delta_{j p} \xi_{k}+\delta_{k p} \xi_{j}\right)\right) G . \\
\partial_{\xi_{j}} \partial_{\xi_{k}} \partial_{\xi_{p}} \partial_{\xi_{q}} G=\left(\frac{16 u^{4} v^{4}}{(u+v)^{4}} \xi_{j} \xi_{k} \xi_{p} \xi_{q}+\frac{4 u^{2} v^{2}}{(u+v)^{2}}\left(\delta_{j k} \delta_{p q}+\delta_{j p} \delta_{k q}+\delta_{k p} \delta_{j q}\right)\right. \\
\left.\quad-\frac{8 u^{3} v^{3}}{(u+v)^{3}}\left(\delta_{j q} \xi_{k} \xi_{p}+\delta_{k q} \xi_{j} \xi_{p}+\delta_{p q} \xi_{j} \xi_{k}+\delta_{j k} \xi_{p} \xi_{q}+\delta_{j p} \xi_{k} \xi_{q}+\delta_{k p} \xi_{j} \xi_{q}\right)\right) G .
\end{gathered}
$$


So for (a),

$$
\sigma\left(\partial_{j} \partial_{k}, I, \partial_{p} \partial_{q}, I, u, v, x, \xi\right)=\xi_{j} \xi_{k} \xi_{p} \xi_{q} G
$$

for (b),

$$
\sigma\left(\partial_{j}, \partial_{k}, \partial_{p} \partial_{q}, I, u, v, x, \xi\right)=-i \xi_{j} \xi_{p} \xi_{q} P\left(\partial_{k}, u,-i \partial_{\xi}\right) G=\frac{\xi_{j} \xi_{p} \xi_{q}}{2 u} \partial_{\xi_{k}} G=-\frac{v}{u+v} \xi_{j} \xi_{k} \xi_{p} \xi_{q} G .
$$

for (c),

$$
\begin{aligned}
\sigma\left(\partial_{j}, \partial_{k}, \partial_{p}, \partial_{q}, u, v, x, \xi\right)=-\xi_{j} \xi_{p} P\left(\partial_{k}, u,-i \partial_{\xi}\right) P\left(\partial_{q}, v,-i \partial_{\xi}\right) G=\frac{\xi_{j} \xi_{p}}{4 u v} \partial_{\xi_{k}} \partial_{\xi_{q}} G \\
=\left(\frac{u v}{(u+v)^{2}} \xi_{j} \xi_{k} \xi_{p} \xi_{q}-\frac{1}{2(u+v)} \delta_{k q} \xi_{j} \xi_{p}\right) G .
\end{aligned}
$$

for $(d)$,

$$
\begin{aligned}
& \sigma\left(I, \partial_{j} \partial_{k}, \partial_{p} \partial_{q}, I, u, v, x, \xi\right)=-\xi_{p} \xi_{q} P\left(\partial_{j} \partial_{k}, u,-i \partial_{\xi}\right) G=\left(\frac{\xi_{p} \xi_{q}}{4 u^{2}} \partial_{\xi_{j}} \partial_{\xi_{k}}+\frac{\xi_{p} \xi_{q} \delta_{j k}}{2 u}\right) G \\
= & \frac{\xi_{p} \xi_{q}}{4 u^{2}}\left(\frac{4 u^{2} v^{2}}{(u+v)^{2}} \xi_{j} \xi_{k}-\frac{2 u v}{u+v} \delta_{j k}\right) G+\frac{\xi_{p} \xi_{q} \delta_{j k}}{2 u} G=\frac{v^{2}}{(u+v)^{2}} \xi_{j} \xi_{k} \xi_{p} \xi_{q} G+\frac{1}{2(u+v)} \delta_{j k} \xi_{p} \xi_{q} G .
\end{aligned}
$$

for (e),

$$
\begin{aligned}
\sigma\left(I, \partial_{j} \partial_{k}, \partial_{p}, \partial_{q}, u, v, x, \xi\right)= & i \xi_{p} P\left(\partial_{j} \partial_{k}, u,-i \partial_{\xi}\right) P\left(\partial_{q}, v,-i \partial_{\xi}\right) G \\
= & \left(\frac{\xi_{p}}{8 u^{2} v} \partial_{\xi_{j}} \partial_{\xi_{k}} \partial_{\xi_{q}}+\frac{\xi_{p} \delta_{j k}}{4 u v} \partial_{\xi_{q}}\right) G \\
=-\frac{u v^{2}}{(u+v)^{3}} \xi_{j} \xi_{k} \xi_{p} \xi_{q} G+ & \frac{v}{2(u+v)^{2}}\left(\delta_{j k} \xi_{p} \xi_{q}+\delta_{j q} \xi_{k} \xi_{p}+\delta_{k q} \xi_{j} \xi_{p}\right) G-\frac{1}{2(u+v)} \delta_{j k} \xi_{p} \xi_{q} G
\end{aligned}
$$

and finally for (f),

$$
\begin{aligned}
\sigma & \left(I, \partial_{j} \partial_{k}, I, \partial_{p} \partial_{q}, u, v, x, \xi\right)=P\left(\partial_{j} \partial_{k}, u, i \partial_{\xi}\right) P\left(\partial_{p} \partial_{q}, v, i \partial_{\xi}\right) G \\
= & \left(\frac{1}{4 u^{2}} \partial_{\xi_{j}} \partial_{\xi_{k}}+\frac{\delta_{j k}}{2 u}\right)\left(\frac{1}{4 v^{2}} \partial_{\xi_{p}} \partial_{\xi_{q}}+\frac{\delta_{p q}}{2 v}\right) G \\
= & \left(\frac{1}{16 u^{2} v^{2}} \partial_{\xi_{j}} \partial_{\xi_{k}} \partial_{\xi_{p}} \partial_{\xi_{q}}+\frac{\delta_{j k}}{8 u v^{2}} \partial_{\xi_{p}} \partial_{\xi_{q}}+\frac{\delta_{p q}}{8 u^{2} v} \partial_{\xi_{j}} \partial_{\xi_{k}}+\frac{\delta_{j k} \delta_{p q}}{4 u v}\right) G \\
= & \left(\frac{u^{2} v^{2}}{(u+v)^{4}} \xi_{j} \xi_{k} \xi_{p} \xi_{q}+\frac{1}{4(u+v)^{2}}\left(\delta_{j k} \delta_{p q}+\delta_{j p} \delta_{k q}+\delta_{k p} \delta_{j q}\right)\right. \\
& \left.\quad-\frac{u v}{2(u+v)^{3}}\left(\delta_{j q} \xi_{k} \xi_{p}+\delta_{k q} \xi_{j} \xi_{p}+\delta_{p q} \xi_{j} \xi_{k}+\delta_{j k} \xi_{p} \xi_{q}+\delta_{j p} \xi_{k} \xi_{q}+\delta_{k p} \xi_{j} \xi_{q}\right)\right) G \\
& +\left(\frac{u}{2(u+v)^{2}} \delta_{j k} \xi_{p} \xi_{q}-\frac{1}{4(u+v) v} \delta_{j k} \delta_{p q}+\frac{v}{2(u+v)^{2}} \xi_{j} \xi_{k} \delta_{p q}-\frac{1}{4 u(u+v)} \delta_{j k} \delta_{p q}+\frac{1}{4 u v} \delta_{j k} \delta_{p q}\right) G \\
& =\left(\frac{u^{2} v^{2}}{(u+v)^{4}} \xi_{j} \xi_{k} \xi_{p} \xi_{q}+\frac{1}{4(u+v)^{2}}\left(\delta_{j k} \delta_{p q}+\delta_{j p} \delta_{k q}+\delta_{k p} \delta_{j q}\right)+\frac{u}{2(u+v)^{2}} \delta_{j k} \xi_{p} \xi_{q}\right. \\
& \left.+\frac{v}{2(u+v)^{2}} \xi_{j} \xi_{k} \delta_{p q}-\frac{u v}{2(u+v)^{3}}\left(\delta_{j q} \xi_{k} \xi_{p}+\delta_{k q} \xi_{j} \xi_{p}+\delta_{p q} \xi_{j} \xi_{k}+\delta_{j k} \xi_{p} \xi_{q}+\delta_{j p} \xi_{k} \xi_{q}+\delta_{k p} \xi_{j} \xi_{q}\right)\right) G .
\end{aligned}
$$


Now we just need to substitute these results for $\sigma$ into (5.16) to obtain the expressions for $u_{s}$ given in Theorem 2 (a)-(f). This is done more efficiently by looking at each type of term which occurs in $\sigma$ and plugging it into (5.16). Writing $S=s-n / 2$, we have

$$
\begin{aligned}
& \frac{1}{\Gamma(s-n / 2)} \int_{0}^{\infty} \int_{0}^{\infty}(u+v)^{S-r} u^{p} v^{q} G d u d v= \\
& (4 \pi)^{-n / 2}|\xi|^{-2 S+2 r-2 p-2 q-4} \frac{\Gamma(S-r+p+q+2) \Gamma(-S+r-p-1) \Gamma(-S+r-q-1)}{\Gamma(S) \Gamma(-2 S+2 r-p-q-2)} \\
& \frac{S \cdots(S+1+p+q-r)(2 S-1) \cdots(2 S+2+p+q-2 r)}{S \ldots(S+1+p-r) S(s)|\xi|^{-2 S+2 r-2 p-2 q-4}} \\
& \quad
\end{aligned}
$$

where $C(s)$ defined in (1.7), and we assume $r \leq \min \{p, q\}+2$. Hence we obtain $u_{s}\left(\partial^{\alpha}, \partial^{\beta}, \partial^{\delta}, \partial^{\gamma}, x, \xi\right)$ from $\sigma\left(\partial^{\alpha}, \partial^{\beta}, \partial^{\delta}, \partial^{\gamma}, u, v, x, \xi\right)$ by substituting

$$
\begin{array}{ll}
\frac{u^{2} v^{2}}{(u+v)^{4}} G \rightarrow\left(S^{2}+S\right)|\xi|^{n-2 s-4}, & \frac{u v}{(u+v)^{3}} G \rightarrow S|\xi|^{n-2 s-2}, \\
\frac{u v^{2}}{(u+v)^{3}} G \rightarrow\left(2 S^{2}+S-1\right)|\xi|^{n-2 s-4}, & \frac{v}{(u+v)^{2}} G \rightarrow(2 S-1)|\xi|^{n-2 s-2}, \\
\frac{u v}{(u+v)^{2}} G \rightarrow\left(4 S^{2}+2 S-2\right)|\xi|^{n-2 s-4}, & \frac{1}{u+v} G \rightarrow(4 S-2)|\xi|^{n-2 s-2}, \\
\frac{v^{2}}{(u+v)^{2}} G \rightarrow\left(4 S^{2}-2 S\right)|\xi|^{n-2 s-4}, & \frac{1}{(u+v)^{2}} G \rightarrow|\xi|^{n-2 s}, \\
G \rightarrow 4\left(4 S^{2}-1\right)|\xi|^{n-2 s-4} . &
\end{array}
$$

\section{The double decomposition.}

In this Section we complete the proof of Theorem 1. In order to simplify the notation we will restrict to the scalar case with $V=1$. It is straight forward to obtain the vector case by replacing scalars by matrices in the appropriate places. Let $\Omega$ be a coordinate chart of $M$ of small diameter which is identified with a subset of $\mathbb{R}^{n}$, and make a change of coordinates $(x, y) \rightarrow(x, w)$, sending $\Omega \times \Omega$ onto $\Lambda \subset \mathbb{R}^{2 n}$, where $(x, y)$ are product coordinates on $\Omega \times \Omega$ and $w=w(x, y)$ are normal coordinates of $y$ centered at $x$, which vary smoothly with $x$. We define the function $\rho(x, w)$ to give the volume element on $M$;

$$
\rho(x, w) d w=d V(y)=\sqrt{g(y)} d y .
$$

For a function $f$ on $\Omega \times \Omega$, we define $f^{\mathcal{N}}$ on $\Lambda$ by

$$
f(x, y)=f^{\mathcal{N}}(x, w(x, y)) .
$$

and we are interested in understanding (4.19), which is a sum of terms of the form

$$
\frac{1}{\Gamma(s-n / 2)} \iint_{u+v<1} \iint_{\Omega \times \Omega}(u+v)^{s}\left(\partial^{\alpha} h_{i j}(x)\right) E(u, v, x, y)\left(\partial^{\gamma} h_{k \ell}(y)\right) \sqrt{g(x)} \sqrt{g(y)} d x d y d u d v
$$


where

$$
E(u, v, x, y)=A(x)\left(\partial_{x}^{\beta} K\left(e^{-u F}, x, y\right)\right) B(y)\left(\partial_{y}^{\delta} K\left(e^{-v F}, y, x\right)\right) .
$$

Here, the smooth functions $A$ and $B$ are supported in $\Omega$. Set

$$
m=|\beta|+|\delta| .
$$

Step 9: We denote the operator on $\Omega$ with kernel $E(u, v, x, y)$ by $\mathcal{E}_{u, v}$ and the metric-modified symbol of this operator (see $(2.4)$ ) by $\sigma(E, u, v, x, \xi)$, so

$$
\sigma(E, u, v, x, \xi)=\int_{\mathbb{R}^{n}} e^{i w \cdot \xi} E^{\mathcal{N}}(u, v, x, w) d w
$$

and for $f \in C^{\infty}(\Omega)$ compactly supported,

$$
\left(\mathcal{E}_{u, v} f\right)(x)=\int_{\Omega} E(u, v, x, y) f(y) \sqrt{g(y)} d y=\frac{1}{(2 \pi)^{n}} \int_{\mathbb{R}^{n}} \int_{\Omega} \sigma(E, u, v, x, \xi) e^{-i w \cdot \xi} f^{\mathcal{N}}(x, w) \rho(x, w) d w d \xi
$$

Steps 14, 15: We will see in Lemma 6.3, that

$$
|\sigma(E, u, v, x, \xi)|=O\left((u+v)^{-n / 2-m}\right), \quad \text { as } u, v \rightarrow 0
$$

Hence

$$
\left(\mathcal{E}_{u, v} f\right)(x)=O\left((u+v)^{-n / 2-m}\right), \quad \text { as } u, v \rightarrow 0,
$$

and when $\Re s>n / 2+m-2$, we can interchange the $(u, v)$ integration in (6.1) with the $x$ integration and conclude that (6.1) equals

$$
\int_{\Omega}\left(\partial^{\alpha} h_{i j}\right)\left(\mathcal{W}_{s} \partial^{\gamma} h_{k \ell}\right) \sqrt{g} d x
$$

where $\mathcal{W}_{s}$ is the operator

$$
\frac{1}{\Gamma(s-n / 2)} \iint_{u+v<1}(u+v)^{s} \mathcal{E}_{u, v} d u d v
$$

which has symbol

$$
\sigma_{s}(x, \xi):=\frac{1}{\Gamma(s-n / 2)} \iint_{u+v<1}(u+v)^{s} \sigma(E, u, v, x, \xi) d u d v
$$

Our aim is to show the following Lemma.

Lemma 6.1. $\sigma_{s}(x, \xi)$ is entire in $s$ and has the form $U_{s}+V_{s}$ where $U_{s}$ has degree $n-2 s-4+m, V_{s}$ has degree -2 , and $U_{s}$ and $V_{s}$ are meromorphic with simple poles in $n / 2+\mathbb{N}^{+}$.

This completes the proof of Theorem 1, since we can now write (6.5) in the form

$$
\int_{\Omega} h_{i j}\left(\mathcal{T}_{s} h_{k \ell}\right) \sqrt{g} d x
$$


where $\mathcal{T}_{s}$ is the sum of polyhomogeneous $\Psi$ DOs of degrees $n-2 s$ and $4-m-2 \leq 2$. We pause to outline the strategy to prove Lemma 6.1. It is convenient to make the change of variables

$$
t=u+v, \quad \tau=u /(u+v),
$$

so

$$
u=t \tau, \quad v=t(1-\tau) .
$$

Write

$$
H(t, \tau, x, y)=E(t \tau, t(1-\tau), x, y)
$$

so

$$
\sigma_{s}(x, \xi)=\frac{1}{\Gamma(s-n / 2)} \int_{0}^{1} \int_{0}^{1} t^{s+1} \sigma(H, t, \tau, x, \xi) d \tau d t .
$$

By (4.8), we see that

$$
H(t, \tau, x, y)=t^{-n-m}(\tau(1-\tau))^{-n / 2-m} e^{-d^{2}(x, y) /(4 t \tau(1-\tau))} P(t, \tau, x, y)
$$

where

$$
P(t, \tau, x, y) \in C^{\infty}\left([0,1]^{2} \times \Omega^{2}\right) .
$$

Steps 12, 13: We make a Taylor expansion of $P(t, \tau, x, y)=P^{\mathcal{N}}(t, \tau, x, w)$ in $w$. The $L^{\text {th }}$ order remainder term has order $|w|^{L}$, and in Lemma 6.2 we see that it contributes a symbol of order $-L+C$ to $\sigma_{s}(x, \xi)$, where $C=C(m, n, s)$ is independent of $L$. The consequence of this is that we can ignore the Taylor remainder term and prove the result when $P(u, v, x, y)$ is replaced by a Taylor polynomial. Step 16: Doing this and carrying out the $\tau$ integral in (6.9), we find in Lemma 6.4 that $\sigma_{s}(x, \xi)$ is a finite sum of terms of the form

$$
\xi^{\nu} \int_{0}^{1} t^{s+j} Q\left(t,|\xi|^{2} t, x\right) d t
$$

where $Q(t, T, x)$ is smooth on $[0,1] \times[0, \infty] \times \Omega$ (including the point $T=+\infty$ ), and vanishes at $T=+\infty$. We show in Lemma 6.5, that such a symbol is entire in $s$. Making a Talyor expansion around $t=0$ and $T=\infty$ enables us to obtain the polyhomogeneous expansions of $U_{s}$ and $V_{s}$. Terms of increasing power in $t$ give terms of decreasing order for $U_{s}$, while terms of decreasing power in $T$ give terms of decreasing order for $V_{s}$. We proceed to carry out this plan.

Proof of Lemma 6.1.

Lemma 6.2. Suppose that $\sigma_{s}$ is given by (6.9) where $H$ is given by (6.10) and $P$ satisfies (6.11). Fix $s \in \mathbb{C}$, and $k \in \mathbb{N}$, and suppose

$$
P^{\mathcal{N}}(t, \tau, x, w)=O\left(|w|^{L}\right)
$$

where

$$
L>k+(n-2 \Re s-2)_{+}+2 m-2 .
$$


Then $\sigma_{s}(x, \xi)$ is a symbol of order $-k$.

This lemma is proved later on. It is a standard fact in the theory of $\Psi$ DOs that given a a sequence $\sigma_{j}(x, \xi)$ of symbols on $\Omega$ with $s_{j}(x, \xi)$ homogeneous of degree $\mu-j$, then there exists a symbol $\sigma(x, \xi)$ with symbol expansion

$$
\sum_{j=1}^{\infty} \sigma_{j}(x, \xi)
$$

Moreover, if each term $\sigma_{j}$ depends analytically on a parameter $s$, then $\sigma$ can be chosen to depend analytically on $s$. This combined with Lemma 6.2 enables us to restrict attention to the case when $P^{\mathcal{N}}(u, v, x, w)$ is replaced by its Taylor polynomial in $w$ of degree $L$;

$$
P_{L}(t, \tau, x, w):=\sum_{|\mu| \leq L} P_{\mu}(t, \tau, x) w^{\mu}
$$

Steps 12, 13: Set

$$
H_{L}(t, \tau, x, w)=t^{-n-m}(\tau(1-\tau))^{-n / 2-m} e^{-|w|^{2} /(4 t \tau(1-\tau))} \sum_{|\mu| \leq L} P_{\mu}(t, \tau, x) w^{\mu} .
$$

Step 15: Then

$$
\begin{aligned}
\sigma\left(H_{L}, t, \tau, x, \xi\right) & =\int_{\mathbb{R}^{n}} e^{i w \cdot \xi} H_{L}(t, \tau, x, w) d w \\
& =t^{-n-m}(\tau(1-\tau))^{-n / 2-m} \sum_{|\mu| \leq L} P_{\mu}(t, \tau, x) \int_{\mathbb{R}^{n}} e^{i w \cdot \xi} w^{\mu} e^{-|w|^{2} /(4 t \tau(1-\tau))} d w \\
& =(4 \pi)^{n / 2} t^{-n / 2-m}(\tau(1-\tau))^{-m} \sum_{|\mu| \leq L} P_{\mu}(t, \tau, x)\left(-i \partial_{\xi}\right)^{\mu} e^{-|\xi|^{2} t \tau(1-\tau)} \\
& =t^{-n / 2-m}(\tau(1-\tau))^{-m} e^{-|\xi|^{2} t \tau(1-\tau)} \sum_{|\mu| \leq L} P_{\mu}(t, \tau, x) \sum_{\substack{|\nu| \leq|\mu| \\
|\mu+\nu| \text { even }}} c_{\mu \nu}(t \tau(1-\tau))^{|\mu+\nu| / 2} \xi^{\nu},
\end{aligned}
$$

where $c_{\mu \nu}$ are constants. The problem with this expression is the presence of the non-integrable factor

$$
(\tau(1-\tau))^{-m}
$$

which may not be neutralized by the factor

$$
(\tau(1-\tau))^{(|\mu+\nu|) / 2}
$$

when $|\nu|$ is small. However, the following lemma shows that this is not an issue after the terms are summed up. We see from (6.14) that we can write $\sigma\left(H_{L}, t, \tau, x, \xi\right)$ in the form

$$
\sigma\left(H_{L}, t, \tau, x, \xi\right)=t^{-n / 2-m} e^{-|\xi|^{2} t \tau(1-\tau)} \sum_{|\nu| \leq L} R_{L, \nu}(t, \tau, x) \xi^{\nu}
$$


Lemma 6.3. If $L \geq 3 m$, then

$$
R_{L, \nu}(t, \tau, x) \in C^{\infty}([0,1] \times[0,1] \times \Omega) .
$$

This lemma is proved later on. We now need to improve our understanding of the dependence of $\sigma\left(H_{L}, t, \tau, x, \xi\right)$ on $t$. Indeed, Paying closer attention to what happens when we differentiate the heat kernel, we see that (6.13) can be improved to

$$
P_{\mu}(t, \tau, x)=O\left(t^{(m-|\mu|)_{+} / 2}\right),
$$

and so from (6.14),

$$
R_{L, \nu}(t, \tau, x)=O\left(t^{(m+|\nu|) / 2}\right) .
$$

Hence writing

$$
\lceil r\rceil:=\inf \{q \in \mathbb{Z}: q \geq r\}
$$

we see that

$$
\tilde{R}_{L, \nu}(t, \tau, x):=t^{-\lceil(m+|\nu|) / 2\rceil} R_{L, \nu}(t, \tau, x) \in C^{\infty}\left([0,1]^{2} \times \Omega\right) .
$$

Now up to a term of order $-L+C$,

$$
\sigma_{s}(x, \xi)=\frac{1}{\Gamma(s-n / 2)} \sum_{|\nu| \leq L} \xi^{\nu} \int_{0}^{1} \int_{0}^{1} t^{s-n / 2+\lceil(|\nu|-m) / 2\rceil+1} e^{-|\xi|^{2} t \tau(1-\tau)} \tilde{R}_{L, \nu}(t, \tau, x) d \tau d t .
$$

It is convenient to compactify the interval $[0, \infty)$ by including the point $+\infty$. The differentiable structure at $+\infty$ is given by the coordinates $\phi:(0,+\infty] \rightarrow[0,+\infty)$,

$$
\phi(T)= \begin{cases}1 / T & T<+\infty \\ 0 & T=+\infty\end{cases}
$$

Lemma 6.4. If

$$
R(t, \tau, x) \in C^{\infty}([0,1] \times[0,1] \times \Omega) .
$$

then

$$
Q(t, T, x):=\int_{0}^{1} e^{-T \tau(1-\tau)} R(t, \tau, x) d \tau
$$

is smooth on $[0,1] \times[0,+\infty] \times \Omega$ and vanishes at $T=+\infty$.

This lemma is proved later on. We need one more result to complete the proof of Lemma 6.1.

Lemma 6.5. Suppose $Q(t, T, x)$ is smooth on $[0,1] \times[0,+\infty] \times \Omega$ and vanishes at $T=+\infty$. Then

$$
\sigma(s, x, \xi):=\int_{0}^{1} t^{s-n / 2} Q\left(t,|\xi|^{2} t, x\right) d t
$$

is a symbol of order -2 on the set $\Re s>n / 2-1$, which extends analytically to a meromorphic function for $s \in \mathbb{C}$, and

$$
\frac{1}{\Gamma(s-n / 2+1)} \sigma(s, x, \xi)
$$


is smooth in $(s, x, \xi)$ and entire in s. Moreover,

$$
\sigma(s, x, \xi)=U_{s}(x, \xi)+V_{s}(x, \xi)
$$

where

$$
\frac{1}{\sin (\pi s)} U_{s}(x, \xi), \quad \frac{1}{\sin (\pi s)} V_{s}(x, \xi),
$$

are polyhomogeneous of degrees $n-2 s-2$ and -2 respectively, smooth in $(s, x, \xi)$ and entire in $s$. The symbol expansions of $U_{s}$ and $V_{s}$ can be computed in terms of the Taylor series of $Q$. Indeed, if

$$
\begin{aligned}
& Q(t, T, x) \sim \sum_{k=0}^{\infty} t^{k} q_{k}(T, x), \quad t \rightarrow 0, \\
& Q(t, T, x) \sim \sum_{\ell=0}^{\infty} T^{-\ell-1} q^{\ell}(t, x), \quad T \rightarrow \infty
\end{aligned}
$$

then as $|\xi| \rightarrow \infty$,

$$
\begin{aligned}
& U_{s}(x, \xi) \sim \sum_{k=0}^{\infty} u_{s, k}(x)|\xi|^{n-2 s-2-2 k}, \quad \text { where } \quad u_{s, k}(x)=\int_{0}^{\infty} T^{s-n / 2+k} q_{k}(T, x) d T \\
& V_{s}(x, \xi) \sim \sum_{k=0}^{\infty} v_{s, k}(x)|\xi|^{-2-2 k}, \quad \text { where } \quad v_{s, k}(x)=\int_{0}^{1} t^{s-n / 2-k-1} q^{k}(t, x) d t .
\end{aligned}
$$

We apply Lemmas 6.4 and 6.5 to (6.15). In order to apply Lemma 6.5 we replace $s$ by $s+\lceil(|\nu|-$ $m) / 2\rceil+1$ and deduce that $\sigma_{s}(x, \xi)$ is analytic for $s>n / 2+m / 2-2$, and is a sum of polyhomogeneous symbols of degrees -2 and $n-2 s+m-4$ which are meromorphic in $s$ with poles in $n / 2+\mathbb{N}^{+}$. Since $m$ is an integer between 0 and 4 , this completes the proof of Lemma 6.1.

The rest of this paper is devoted to proving Lemmas 6.2, 6.3, 6.4 and 6.5.

Proof of Lemma 6.2. We consider the kernel $K_{s}$ with symbol $\sigma_{s}$. This is given by

$$
\begin{aligned}
K_{s}(x, w): & =\frac{1}{\Gamma(s-n / 2)} \int_{0}^{1} \int_{0}^{1} t^{s+1} H^{\mathcal{N}}(t, \tau, x, w) d w \\
& =\frac{1}{\Gamma(s-n / 2)} \int_{0}^{1} \int_{0}^{1} t^{s+1-n-m}(\tau(1-\tau))^{-n / 2-m} e^{-|w|^{2} /(4 t \tau(1-\tau))} P(t, \tau, x, w) d \tau d t .
\end{aligned}
$$

Now suppose $\kappa$ is a multiindex and $|\kappa|=k$. Then

$$
\begin{aligned}
& \left|\partial_{w}^{\kappa} \partial_{x}^{\nu} K_{s}(x, w)\right| \\
& \quad=\left|\frac{1}{\Gamma(s-n / 2)} \int_{0}^{1} \int_{0}^{1} t^{s+1-n-m}(\tau(1-\tau))^{-n / 2-m} \partial_{w}^{\kappa}\left(e^{-|w|^{2} /(4 t \tau(1-\tau))} \partial_{x}^{\nu} P(t, \tau, x, w)\right) d \tau d t\right|
\end{aligned}
$$


Using Leibnitz rule for differentiation, we see that this is bounded by a linear combination of terms of the following form where $\ell$ is in the range $0 \leq \ell \leq k$.

$$
|w|^{L+2 \ell-k} \int_{0}^{1} \int_{0}^{1} t^{\Re s+1-n-m-\ell}(\tau(1-\tau))^{-n / 2-m-\ell} e^{-|w|^{2} /(4 t \tau(1-\tau))} d \tau d t,
$$

and setting $T=|w|^{-2} t$, this has the form

$$
|w|^{L-k+2 \Re s-2 n-2 m+4} \int_{0}^{|w|^{-2}} T^{\Re s+1-n-m-\ell} \int_{0}^{1}(\tau(1-\tau))^{-n / 2-m-\ell} e^{-1 /(4 T \tau(1-\tau))} d \tau d T .
$$

Now

$$
\int_{0}^{1}(\tau(1-\tau))^{-n / 2-m-\ell} e^{-1 /(4 T \tau(1-\tau))} d \tau= \begin{cases}O\left(e^{-1 /(2 T)}\right), & T \rightarrow 0 \\ O\left(T^{n / 2+m+\ell-1}\right) & T \rightarrow \infty\end{cases}
$$

so we conclude that

$$
\left|\partial_{w}^{\kappa} \partial_{x}^{\nu} K_{s}(x, w)\right| \leq C(n, s)|w|^{L-k+2 \Re s-2 n-2 m+4}\left(1+|w|^{n-2 \Re s-2} \log (c /|w|)\right),
$$

where $c$ is a constant larger than the diameter of $M$. This implies that $\partial_{w}^{\kappa} \partial_{x}^{\nu} K_{s}(x, w)$ is integrable when

$$
L>k+(n-2 \Re s-2)_{+}+2 m-2 .
$$

Now suppose that (6.16) holds for some integer $k \geq 0$. Then

$$
\sigma_{s}(x, \xi)=\int_{\mathbb{R}^{n}} e^{i w \cdot \xi} K_{s}(x, w) d w
$$

and

$$
\partial_{x}^{\nu} \partial_{\xi}^{\mu} \sigma_{s}(x, \xi)=\int_{\mathbb{R}^{n}} e^{i w \cdot \xi}(i w)^{\mu} \partial_{x}^{\nu} K_{s}(x, w) d w .
$$

For fixed $\xi$, there exists a $j$ such that

$$
\left|\xi_{j}\right| \geq \frac{|\xi|}{\sqrt{n}}
$$

Then define the multiindex $\kappa$ by

$$
\left\{\begin{array}{l}
\kappa_{\ell}=0 \\
\kappa_{j}=k+|\mu| .
\end{array}\right.
$$

so

$$
\left|\xi^{\kappa}\right| \geq C|\xi|^{k+|\mu|}, \quad \text { where } \quad C=C(n, k, \mu)>0
$$

Since

$$
e^{i w \cdot \xi}=\frac{1}{(i \xi)^{\kappa}}\left(\partial_{w}^{\kappa} e^{i w \cdot \xi}\right)
$$

we can integrate by parts to obtain

$$
\partial_{x}^{\nu} \partial_{\xi}^{\mu} \sigma_{s}(x, \xi)=\frac{1}{(-i \xi)^{\kappa}} \int_{\mathbb{R}^{n}} e^{i w \cdot \xi} \partial_{w}^{\kappa} \partial_{x}^{\nu}\left((i w)^{\mu} K_{s}(x, w)\right) d \tau d t d w .
$$


Replacing $L$ and $k$ in (6.16) by $L+|\mu|$ and $k+|\mu|$, and choosing $R$ greater than the diameter of $M$,

$$
\left|\partial_{x}^{\nu} \partial_{\xi}^{\mu} \sigma_{s}(x, \xi)\right| \leq \frac{C(n, s, k, \mu)}{|\xi|^{k+|\mu|}} \int_{|w| \leq R}|w|^{L-k+2 \Re s-2 n-2 m+4}\left(1+|w|^{n-2 \Re s-2} \log (c /|w|)\right) d w .
$$

The integral on the right is finite when (6.16) holds. This completes the proof of Lemma 6.2.

Proof of Lemma 6.3. We will start by listing the relevant definitions. Set

$$
\Lambda=\left\{(x, w):(x, y) \in \Omega^{2}\right\} \subset \Omega \times \mathbb{R}^{n} .
$$

We have

$$
\begin{gathered}
E(u, v, x, y)=A(x)\left(\partial_{x}^{\beta} K\left(e^{-u F}, x, y\right)\right) B(y)\left(\partial_{y}^{\delta} K\left(e^{-v F}, y, x\right)\right), \\
m=|\beta|+|\delta| .
\end{gathered}
$$

Under the change of coordinates $(u, v) \rightarrow(t, \tau)$ the function $E$ transforms to $H$ which has the form,

$$
H(t, \tau, x, y)=t^{-n-m}(\tau(1-\tau))^{-n / 2-m} e^{-d^{2}(x, y) /(4 t \tau(1-\tau))} P(t, \tau, x, y) .
$$

where

$$
P(t, \tau, x, y) \in C^{\infty}\left([0,1]^{2} \times \Omega^{2}\right) .
$$

Taking the $L^{t h}$ order Taylor polynomial of $P^{\mathcal{N}}(t, \tau, x, w)$ in $w$ we have

$$
P_{L}(t, \tau, x, w)=\sum_{|\mu| \leq L} P_{\mu}(t, \tau, x) w^{\mu}
$$

The part of $H^{\mathcal{N}}$ corresponding to $P_{L}$ is $H_{L}$,

$$
H_{L}(t, \tau, x, w)=t^{-n-m}(\tau(1-\tau))^{-n / 2-m} e^{-|w|^{2} /(4 t \tau(1-\tau))} \sum_{|\mu| \leq L} P_{\mu}(t, \tau, x) w^{\mu} .
$$

Taking the Fourier transform in $w$, we get the symbol $\sigma\left(H_{L}\right)$,

$$
\begin{aligned}
\sigma\left(H_{L}, t, \tau, x, \xi\right) & =t^{-n / 2-m} e^{-|\xi|^{2} t \tau(1-\tau)}(\tau(1-\tau))^{-m} \sum_{|\mu| \leq L} P_{\mu}(t, \tau, x) \sum_{\substack{|\nu| \leq|\mu| \\
|\nu+\mu| \text { even }}} c_{\mu \nu}(t \tau(1-\tau))^{|\mu+\nu| / 2} \xi^{\nu} \\
& =t^{-n / 2-m} e^{-|\xi|^{2} t \tau(1-\tau)} \sum_{|\nu| \leq L} R_{L, \nu}(t, \tau, x) \xi^{\nu}
\end{aligned}
$$

Now we start the proof of Lemma 6.3 by showing that if $L>3 m$ then

$$
R_{L, \nu}(t, \tau, x) \in C^{\infty}([0,1] \times(0,1] \times \Omega) .
$$

This is equivalent to showing that

$$
t^{n / 2+m} e^{|\xi|^{2} t \tau(1-\tau)} \sigma\left(H_{L}, t, \tau, x, \xi\right) \in C^{\infty}\left([0,1] \times(0,1] \times \Omega \times \mathbb{R}^{n}\right) .
$$


The key here is to write

$$
\begin{aligned}
& E^{\mathcal{N}}(t, \tau, x, \xi) \\
& =\sum_{|\mu+\nu+\iota+\kappa| \leq m} C_{\mu, \nu, \iota, \kappa} \partial_{x}^{\mu} \partial_{w}^{\nu}\left(S_{\mu, \nu, \iota, \kappa}(x, w) \quad\left(\partial_{x}^{\iota} \partial_{w}^{\kappa} K^{\mathcal{N}}\left(e^{-t \tau F}, x, w\right)\right) \quad K^{\mathcal{N}}\left(e^{-t(1-\tau) F}, w, x\right)\right),
\end{aligned}
$$

where

$$
S_{\mu, \nu, \iota, \kappa}(x, w) \in C^{\infty}\left([0,1]^{2} \times \Lambda\right),
$$

and the partial derivatives are taken with respect to the $(x, w)$ coordinates. This is accomplished by first changing variables from $(x, y)$ to $(x, w)$, and then using the product rule to take the derivatives off the factor $\left.K^{\mathcal{N}}\left(e^{-v F}, w, x\right)\right)$. Now from $(4.8)$,

$$
K^{\mathcal{N}}\left(e^{-u F}, x, w\right)=(4 \pi u)^{-n / 2} e^{-|w|^{2} / 4 u} b^{\mathcal{N}}(u, x, w) .
$$

Hence

$$
E^{\mathcal{N}}(t, \tau, x, \xi)=\sum_{|\nu| \leq m} t^{-n-m+|\nu|} \tau^{-n / 2-m+|\nu|}(1-\tau)^{-n / 2} \partial_{w}^{\nu}\left(e^{-|w|^{2} /(4 t \tau(1-\tau))} X_{\nu}(t, \tau, x, w)\right)
$$

where

$$
X_{\nu} \in C^{\infty}\left([0,1]^{2} \times \Lambda\right) .
$$

Denote the $L^{\text {th }}$ order Taylor polynomial of $X$ in $w$ by

$$
X_{\nu}^{L}(t, \tau, x, w)=\sum_{|\mu| \leq L} X_{\nu, \mu}(t, \tau, x) w^{\mu} .
$$

Now replacing $X_{\nu}$ in (6.20) by $X_{\nu}^{L}$ we form $H^{L}$,

$$
H^{L}(t, \tau, x, w):=\sum_{|\nu| \leq m} t^{-n-m+|\nu|} \tau^{-n / 2-m+|\nu|}(1-\tau)^{-n / 2} \partial_{w}^{\nu}\left(e^{-|w|^{2} /(4 t \tau(1-\tau))} X_{\nu}^{L}(t, \tau, x, w)\right) .
$$

Comparing this with $H_{L}$, we find that only terms with degree close to $L$ contribute to the difference

$$
H^{L}(t, \tau, x, w)-H_{L}(t, \tau, x, w)
$$

Indeed, this difference has the form

$$
t^{-n-m}(\tau(1-\tau))^{-n / 2-m} e^{-|w|^{2} /(4 t \tau(1-\tau))} \sum_{L-m \leq|\theta| \leq L+m} Y_{\theta}(t, \tau, x) w^{\theta},
$$

where

$$
Y_{\theta}(t, \tau, x) \in C^{\infty}\left([0,1]^{2} \times \Omega\right) .
$$

Then computing the symbol by following (6.14),

$$
\sigma\left(H^{L}, t, \tau, x, \xi\right)-\sigma\left(H_{L}, t, \tau, x, \xi\right)
$$


is a linear combination of terms of the form

$$
t^{-n / 2-m}(\tau(1-\tau))^{-m} \sum_{L-m \leq|\theta| \leq L+m} Y_{\theta}(t, \tau, x)\left(-i \partial_{\xi}\right)^{\theta} e^{-|\xi|^{2} t \tau(1-\tau)}
$$

and this is a linear combination of terms of the form

$$
t^{-n / 2}(t \tau(1-\tau))^{-m+(|\theta|+|\nu|) / 2} e^{-|\xi|^{2} t \tau(1-\tau)} Y_{\theta}(t, \tau, x) \xi^{\nu},
$$

where

$$
L-m \leq|\theta| \leq L+m, \quad(|\theta|+|\nu|) / 2 \in \mathbb{N} .
$$

When $L \geq 3 m$, we conclude that $-m+(|\theta|+|\nu|) / 2 \geq 0$ and so

$$
t^{n / 2+m} e^{|\xi|^{2} t \tau(1-\tau)}\left(\sigma\left(H^{L}, t, \tau, x, \xi\right)-\sigma\left(H_{L}, t, \tau, x, \xi\right)\right) \in C^{\infty}\left([0,1]^{2} \times \Omega \times \mathbb{R}^{n}\right) .
$$

However, again following (6.14), we find that

$$
\sigma\left(H^{L}, t, \tau, x, \xi\right)
$$

is a linear combination of terms of the form

$$
t^{-n / 2-m} \tau^{-m} X_{\nu \mu}(t, \tau, x)(i \xi)^{\nu} e^{-|\xi|^{2} t \tau(1-\tau)}, \quad X_{\nu \mu} \in C^{\infty}\left([0,1]^{2} \times \Omega\right) .
$$

Hence

$$
t^{n / 2+m} e^{|\xi|^{2} t \tau(1-\tau)} \sigma\left(H^{L}, t, \tau, x, \xi\right) \in C^{\infty}\left([0,1] \times(0,1] \times \Omega \times \mathbb{R}^{n}\right) .
$$

which proves (6.18). Interchanging the roles of $\tau$ and $1-\tau$ by taking the derivatives off the factor $\left.K^{\mathcal{N}}\left(e^{-t \tau F}, x, w\right)\right)$ in $(6.19)$, we prove similarly that

$$
t^{n / 2+m} e^{|\xi|^{2} t \tau(1-\tau)} \sigma\left(H_{L}, t, \tau, x, \xi\right) \in C^{\infty}\left([0,1] \times[0,1) \times \Omega \times \mathbb{R}^{n}\right) .
$$

This completes the proof of Lemma 6.3.

Proof of Lemma 6.4. It is clear that since $R$ is smooth on $[0,1]^{2} \times \Omega$,

$$
Q(t, T, x)=\int_{0}^{1} e^{-T \tau(1-\tau)} R(t, \tau, x) d \tau
$$

is smooth on $[0,1] \times[0, \infty) \times \Omega$. The behavior of $Q$ at $T=\infty$ is an application of the method of steepest descent. Indeed,

$$
Q(t, T, x)=\int_{|\tau-1 / 2|<1 / 4} e^{-\tau(1-\tau) T} R(t, \tau, x) d \tau+\int_{|\tau-1 / 2|>1 / 4} e^{-\tau(1-\tau) T} R(t, \tau, x) d \tau
$$

The first term on the right is smooth for $(t, 1 / T, x) \in[0,1] \times[0, \infty) \times \Omega$ and has zero Taylor expansion at $1 / T=0$. For the second term, make the change of variables $\kappa=T \tau(1-\tau)$ to get

$$
\begin{array}{r}
Q(t, T, x)=\frac{1}{T} \int_{0}^{3 T / 16} e^{-\kappa}\left(R\left(t, \frac{1}{2}-\sqrt{\frac{1}{4}-\kappa / T}, x\right)+R\left(t, \frac{1}{2}+\sqrt{\frac{1}{4}-\kappa / T}, x\right)\right) \sqrt{\frac{1}{4}-\kappa / T} d \kappa \\
=\frac{1}{T} \int_{0}^{3 T / 16} e^{-\kappa} f(t, \kappa / T, x) d \kappa
\end{array}
$$


where $f \in C^{\infty}([0,1] \times[0,3 / 16] \times \Omega)$. It is straight forward to show that setting $\varepsilon=1 / T$, this is smooth for $(t, \varepsilon, x) \in[0,1] \times[0, \infty) \times \Omega$ and vanishes at $\varepsilon=0$. This completes the proof of Lemma 6.4.

Proof of Lemma 6.5. We will need some additional notation. We make Taylor expansions of the terms $q_{k}(T, x)$ as $T \rightarrow \infty:$

$$
q_{k}(T, x) \sim \sum_{\ell=0}^{\infty} q_{k}^{\ell}(x) T^{-\ell-1} .
$$

Then naturally the terms in these expansions also occur in the expansions of $q^{\ell}(t, x)$ as $t \rightarrow 0$,

$$
q^{\ell}(t, x) \sim \sum_{k=0}^{\infty} q_{k}^{\ell}(x) t^{k}
$$

We also have a Taylor expansion of $q_{k}(T, x)$ as $T \rightarrow 0$,

$$
q_{k}(T, x) \sim \sum_{m=0}^{\infty} q_{k, m}(x) T^{m}
$$

Set

$$
Q_{K}(t, T, x)=\frac{1}{t^{K}}\left(Q(t, T, x)-\sum_{k=0}^{K-1} q_{k}(T, x) t^{k}\right),
$$

so $Q_{k}(t, T, x)$ is smooth up to $t=0$, and as $T \rightarrow \infty$ we have the Taylor expansion

$$
Q_{K}(t, T, x) \sim \sum_{\ell=0}^{\infty} Q_{K}^{\ell}(t, x) T^{-\ell-1}
$$

where

$$
Q_{K}^{\ell}(t, x)=\frac{1}{t^{K}}\left(q^{\ell}(t, x)-\sum_{k=0}^{K-1} q_{k}^{\ell}(x) t^{k}\right) .
$$

We will start by showing how to obtain the asymptotic expansion of $\sigma(s, x, \xi)$ which holds in the $L^{\infty}$ norm. The symbol estimates will be discussed afterwards. Now $\sigma(s, x, \xi)$ converges and is analytic in $s$ for $\Re s>n / 2-1$ and we obtain an analytic continuation by using the Taylor series of $Q(t, T, x)$ at $t=T=0$. Indeed, Taylor expanding $Q(t, T, x)$ at $t=0$,

$$
\sigma(s, x, \xi)=\int_{t=0}^{1} t^{s-n / 2+K} Q_{K}\left(t, t|\xi|^{2}, x\right) d t+\sum_{k=0}^{K-1} \int_{0}^{1} t^{s-n / 2+k} q_{k}\left(|\xi|^{2} t, x\right) d t
$$

The first integral converges for $\Re s>n / 2-1-K$. Changing variables,

$$
\int_{0}^{1} t^{s-n / 2+k} q_{k}\left(|\xi|^{2} t, x\right) d t=|\xi|^{n-2 s-2-2 k} \int_{0}^{|\xi|^{2}} T^{s-n / 2+k} q_{k}(T, x) d T .
$$

Fixing $k<2 L$, we will analyze

$$
\psi_{k}(s, x, \xi):=\int_{0}^{|\xi|^{2}} T^{s-n / 2+k} q_{k}(T, x) d T .
$$


This has an analytic continuation which is seen by making a Taylor expansion at $T=0$. Indeed,

$$
\begin{aligned}
\psi_{k}(s, x, \xi)=\int_{0}^{|\xi|^{2}} T^{s-n / 2+K}\left(q_{k}(T, x)-\sum_{m=0}^{K-1} q_{k, m}(x) T^{m}\right) d T & \\
& +\sum_{m=0}^{K-1} \frac{q_{k, m}(x)}{s-n / 2+k+m+1}|\xi|^{2 s-n+2 k+2 m+2} .
\end{aligned}
$$

The integral on the right hand side converges and is analytic in $s$ for $\Re s>n / 2-K-1$, and the other terms are meromorphic with simple poles in the set $n / 2-1-\mathbb{N}^{+}$. This completes the proof that $\sigma(s, x, \xi)$ has a analytic continuation. Furthermore, set

$$
\phi_{k}(s, x, \xi)=\int_{|\xi|^{2}}^{\infty} T^{s-n / 2+k} q_{k}(T, x) d T .
$$

This is convergent for $\Re s<n / 2-k$, and it has an analytic continuation, which can be seen by taking a Taylor series as $T \rightarrow \infty$. Indeed,

$$
\begin{aligned}
\int_{|\xi|^{2}}^{\infty} T^{s-n / 2+k} q_{k}(T, x) d T=\int_{|\xi|^{2}}^{\infty} T^{s-n / 2+k}\left(q_{k}(T, x)\right. & \left.-\sum_{\ell=0}^{L-1} q_{k}^{\ell}(x) T^{-\ell-1}\right) d T \\
& +\sum_{\ell=0}^{L-1} \frac{q_{k}^{\ell}(x)}{s-n / 2+k-\ell}|\xi|^{2 s-n+2 k-2 \ell} .
\end{aligned}
$$

The first integral is convergent for $\Re s<n / 2-k+L$, and is $O\left(|\xi|^{2 \Re s-n+2 k-2 L}\right)$ and the other terms are meromorphic with simple poles in the set $n / 2+\mathbb{N}$. We see that as $|\xi| \rightarrow \infty$,

$$
\int_{|\xi|^{2}}^{\infty} T^{s-n / 2+k} q_{k}(T, x) d T \sim-\sum_{\ell=0}^{\infty} \frac{q_{k}^{\ell}(x)}{s-n / 2+k-\ell}|\xi|^{2 s-n+2 k-2 \ell},
$$

and this asymptotic formula still holds after analytic continuation. Now from this we see that

$$
u_{s, k}(x)=\phi_{k}(s, x, \xi)+\psi_{k}(s, x, \xi)=\int_{0}^{\infty} T^{s-n / 2+k} q_{k}(T, x) d T,
$$

which converges for $n / 2-k-1<\Re s<n / 2-k$, has an analytic continuation to $s \in \mathbb{C}$ with simple poles in $n / 2+\mathbb{N}$, and as $|\xi| \rightarrow \infty$,

$$
\phi_{k}(s, x, \xi) \sim u_{s, k}(x)+\sum_{\ell=0}^{\infty} \frac{q_{k}^{\ell}(x)}{s-n / 2+k-\ell}|\xi|^{2 s-n+2 k-2 \ell},
$$

and from this,

$$
\begin{aligned}
\int_{0}^{1} t^{s-n / 2+k} q_{k}\left(|\xi|^{2} t, x\right) d t=|\xi|^{n-2 s-2-2 k} \phi_{k}(s, x, \xi) & \\
& \sim|\xi|^{n-2 s-2-2 k} u_{s, k}(x)+\sum_{\ell=0}^{\infty} \frac{q_{k}^{\ell}(x)}{s-n / 2+k-\ell}|\xi|^{-2 \ell-2}
\end{aligned}
$$


To obtain the asymptotic behavior of $\sigma(s, x, \xi)$ it remains to compute the asymptotic behavior of

$$
\int_{0}^{1} t^{s-n / 2+K} Q_{K}\left(t, t|\xi|^{2}, x\right) d t
$$

We deal with this by making a Taylor expansion of $Q_{K}(t, T, x)$ as $T \rightarrow \infty$ :

$$
\begin{aligned}
\int_{0}^{1} t^{s-n / 2+K} Q_{K}\left(t,|\xi|^{2} t, x\right) d t & \\
=\int_{0}^{1} t^{s-n / 2+K}\left(Q_{K}\left(t,|\xi|^{2} t, x\right)\right. & \left.-\sum_{\ell=0}^{L-1}|\xi|^{-2 \ell-2} Q_{K}^{\ell}(t, x) t^{-\ell-1}\right) d t \\
& +\sum_{\ell=0}^{L-1}\left(\int_{0}^{1} t^{s-n / 2+K-\ell-1} Q_{K}^{\ell}(t, x) d t\right)|\xi|^{-2 \ell-2}
\end{aligned}
$$

The first integral on the right is $O\left(|\xi|^{-2 L-2}\right)$ provided $\Re s>n / 2-K+L$. Choosing $K=2 L$, we find that it is $O\left(|\xi|^{-2 L-2}\right)$ on $\Re s>n / 2-L$. Putting this together with (6.23) we find that on the set $\Re s>n / 2-L$, as $\xi \rightarrow \infty$,

$$
\begin{aligned}
\sigma(s, x, \xi) \sim \sum_{k=0}^{2 L-1}|\xi|^{n-2 s-2-2 k} u_{s, k}(x) & +\sum_{k=0}^{2 L-1} \sum_{\ell=0}^{L-1} \frac{q_{k}^{\ell}(x)}{s-n / 2+k-\ell}|\xi|^{-2 \ell-2} \\
& +\sum_{\ell=0}^{L-1}\left(\int_{0}^{1} t^{s-n / 2+2 L-\ell-1} Q_{2 L}^{\ell}(t, x) d t\right)|\xi|^{-2 \ell-2}+O\left(|\xi|^{-2 L-2}\right) .
\end{aligned}
$$

We note that

$$
Q_{2 L}^{\ell}(t, x)=\frac{1}{t^{2 L}}\left(q^{\ell}(t, x)-\sum_{k=0}^{2 L-1} q_{k}^{\ell}(x) t^{k}\right),
$$

and so

$$
\begin{aligned}
\sigma(s, x, \xi) & \sim \sum_{k=0}^{2 L-1}|\xi|^{n-2 s-2-2 k} u_{s, k}(x)+\sum_{k=0}^{2 L-1} \sum_{\ell=0}^{L-1} \frac{q_{k}^{\ell}(x)}{s-n / 2+k-\ell}|\xi|^{-2 \ell-2} \\
& +\sum_{\ell=0}^{L-1} \int_{0}^{1} t^{s-n / 2-\ell-1}\left(q^{\ell}(t, x)-\sum_{k=0}^{2 L-1} q_{k}^{\ell}(x) t^{k}\right) d t|\xi|^{-2 \ell-2}+O\left(|\xi|^{-2 L}\right) \\
= & \sum_{k=0}^{2 L-1}|\xi|^{n-2 s-2-2 k} u_{s, k}(x)+\sum_{\ell=0}^{L-1} v_{s, k}(x)|\xi|^{-2 \ell-2}+O\left(|\xi|^{-2 L-2}\right)
\end{aligned}
$$

where $v_{s, k}(x)$ is extended from its domain of convergence by analytic continuation. It remains to show that all the above asymptotic formulas actually hold in the sense of symbols. The key formulas are (6.22) and (6.24). For (6.24), we set

$$
R_{K}^{L}(t, T, x)=Q_{K}(t, T, x)-\sum_{\ell=0}^{L-1} Q_{K}^{\ell}(t, x) T^{-\ell-1},
$$


SO

$$
\left|\partial_{t}^{k} \partial_{T}^{j} \partial_{x}^{\alpha} R_{K}^{L}(t, T, x)\right| \leq C T^{-L-j-1}
$$

and

$$
\partial_{x}^{\alpha} \partial_{\xi}^{\beta} \int_{0}^{1} t^{s-n / 2+K} R_{K}^{L}\left(t,|\xi|^{2} t, x\right) d t
$$

is a sum of terms of the form

$$
\xi^{\gamma} \int_{0}^{1} t^{s-n / 2+K+j}\left(\partial_{x}^{\alpha} \partial_{T}^{j} R_{K}^{L}\right)\left(t,|\xi|^{2} t, x\right) d t
$$

where $|\gamma|=2 j-|\beta|$. This is bounded by

$$
|\xi|^{|\gamma|-2 L-2 j-2} \int_{0}^{1} t^{\Re s-n / 2+K-L-1} d t
$$

which is equal to

$$
C(n, s)|\xi|^{-2 L-2-|\beta|}
$$

provided $\Re s>n / 2-K+L$. This shows that

$$
\int_{0}^{1} t^{s-n / 2+2 L} R_{2 L}^{L}\left(t,|\xi|^{2} t, x\right) d t
$$

is a symbol of order $-2 L-2$ when $\Re s>n / 2-L$.

Similarly, for $(6.22)$, set

$$
r_{k}^{L}(T, x)=q_{k}(T, x)-\sum_{\ell=0}^{L-1} q_{k}^{\ell}(x) T^{-L-1}
$$

SO

$$
\left|\partial_{x}^{\alpha} \partial_{T}^{j} r_{k}^{L}(T, x)\right| \leq C T^{-L-j-1}
$$

Then

$$
\partial_{\xi_{m}} \int_{|\xi|^{2}}^{\infty} T^{s-n / 2+k} r_{k}^{L}(T, x) d T=-2 \xi_{m}|\xi|^{2 s-n+2 k} r_{k}^{L}\left(|\xi|^{2}, x\right)
$$

is a symbol of order $2 \Re s-n+2 k-2 L-1$, and

$$
\left|\partial_{x}^{\alpha} \int_{|\xi|^{2}}^{\infty} T^{s-n / 2+k} r_{k}^{L}(T, x) d T\right| \leq C|\xi|^{2 \Re s-n+2 k-2 L}
$$

It follows that

$$
\phi_{k}(s, x, \xi)-u_{s, k}(x)+\sum_{\ell=0}^{L-1} q_{k}^{\ell}(x)|\xi|^{2 s-n+2 k-2 \ell}=\int_{|\xi|^{2}}^{\infty} T^{s-n / 2+k} r_{k}^{L}(T, x) d T
$$

is a symbol of order $2 \Re s-n+2 k-2 L$, and putting this together, the $O\left(|\xi|^{-2 L-2}\right)$ error in (6.25) is actually a symbol of order $-2 L-2$. This completes the proof of Lemma 6.5. 


\section{References}

[Br] T. Branson, Sharp inequalities, the functional determinant, and the complementary series, Preprint.

[BCY] T. Branson, S. Y. A. Chang and P. Yang, Estimates and extremals for zeta function determinants on four-manifolds, Commun. Math. Phys. 149, (1992), 241-262.

[CGY] S. Y. A. Chang, M. Gursky and P. Yang, An equation of Monge-Ampere type in conformal geometry, and four-manifolds of positive Ricci curvature," Annals of Math., 155 (2002) pp. 1-79.

[CQ] S. Y. A. Chang and J. Qing, Zeta Function Determinants on Manifolds with Boundary, Math. Research Letters 3, 1-17 (1996).

[CY] S. Y. A. Chang and P. Yang, Extremal Metrics of zeta function determinants on 4-manifolds, Annals of Math. 142 (1995) 171-212.

[Ch] I. Chavel, Eigenvalues in Riemannian geometry, Pure and Applied Math. 115, Academic Press, (1984).

[Gi] P. Gilkey, The index theorem and the heat equation. Publish or Perish, Inc., Boston, Mass., (1974).

[GS] V. Guillemin and S. Sternberg, Some remarks on I. M. Gelfand's work, Izrail M. Gelfand, Collected Papers, Vol. I, Springer-Verlag, Berlin, (1987), 831-836.

[Hö] L. Hörmander, The spectral function of an elliptic operator. Acta Math. 121, 193-218 (1968).

[Mo1] C. Morpurgo, Sharp trace inequalities for intertwining operators on $S^{n}$ and $R^{n}$. Internat. Math. Res. Notices (1999), 20, 1101-1117.

[Mo2] C. Morpurgo, Sharp inequalities for functional integrals and traces of conformally invariant operators, Duke Math. Journal, to appear.

[Ok1] K. Okikiolu, Critical metrics for the determinant of the Laplacian in odd dimensions. Ann. of Math. 153 (2001), 471-531.

[Ok2] K. Okikiolu, Critical metrics for the zeta function of a family of scalar Laplacians, Preprint.

[OW] K. Okikiolu and C. Wang, Hessian of the zeta function for the Laplacian on forms. Preprint.

[OPS1] B. Osgood, R. Phillips and P. Sarnak, Extremals of Determinants of Laplacians, J. Funct. Anal. 80 (1988), 148-211.

[OPS2] B. Osgood, R. Phillips and P. Sarnak, Compact isospectral sets of surfaces, J. Funct. Anal. 80 (1988), 212-234.

[OPS3] B. Osgood, R. Phillips and P. Sarnak, Moduli space, heights and isospectral sets of plane domains, Annals of Math. 129 (1989), 293-362.

[ Ri] K. Richardson, Critical points of the determinant of the Laplace operator, Jour. Funct. Anal., 122, 52-83 (1994).

[Se] R. Seeley, Complex Powers of an Elliptic Operator, Proc. Symp. on Singular Integrals, AMS 10, (1967), 131-160.

[Tr] F. Treves, Introduction to pseudodifferential and Fourier integral operators. Vol. 1., Plenum Press, (1980).

Kate Okikiolu

University of California, San Diego

okikiolu@math.ucsd.edu 